\title{
Evolving Fuzzy Rules for Relaxed-Criteria Negotiation
}

\author{
Kwang Mong Sim
}

\begin{abstract}
In the literature on automated negotiation, very few negotiation agents are designed with the flexibility to slightly relax their negotiation criteria to reach a consensus more rapidly and with more certainty. Furthermore, these relaxed-criteria negotiation agents were not equipped with the ability to enhance their performance by learning and evolving their relaxedcriteria negotiation rules. The impetus of this work is designing market-driven negotiation agents (MDAs) that not only have the flexibility of relaxing bargaining criteria using fuzzy rules, but can also evolve their structures by learning new relaxed-criteria fuzzy rules to improve their negotiation outcomes as they participate in negotiations in more e-markets. To this end, an evolutionary algorithm for adapting and evolving relaxed-criteria fuzzy rules was developed. Implementing the idea in a testbed, two kinds of experiments for evaluating and comparing EvEMDAs (MDAs with relaxed-criteria rules that are evolved using the evolutionary algorithm) and EMDAs (MDAs with relaxed-criteria rules that are manually constructed) were carried out through stochastic simulations. Empirical results show that: 1) EvEMDAs generally outperformed EMDAs in different types of e-markets and 2) the negotiation outcomes of EvEMDAs generally improved as they negotiated in more e-markets.
\end{abstract}

Index Terms-Adaptive agent, automated negotiation, evolutionary algorithm, evolutionary computational economics, fuzzy decision controller (FDC), intelligent agent, negotiation agent.

\section{INTRODUCTION}

$\mathbf{N}$ EGOTIATION activities are essential in systems involving the interactions of multiple (artificial or human) agents. In distributed systems, automated negotiation [1] provides a means for agents to resolve differences and conflicting goals, and for controlling and managing resources [2]-[6]. Whereas there is a huge literature on bargaining approaches based on game theory (e.g., [7]-[13]), and automated negotiation for e-commerce (e.g., [14]-[22]), in many of the existing negotiation agents, the decision to reach an agreement is made when the current (counter-)proposals of two negotiating agents coincide. Most of these agents (except for [3], [5], [6], [21], and [22]) were not designed with the flexibility to slightly relax their bargaining terms to reach a consensus more rapidly (and perhaps with more certainty) in the face of intense ne-

Manuscript received August 31, 2007; revised February 29, 2008 and June 8, 2008. First published October 3, 2008; current version published November 20, 2008. This work was supported by a Research Grant from the Hong Kong Research Grant Council's Competitive Earmarked Research Grant under Project HKBU-210906. This paper was recommended by Associate Editor A. G. Skarmeta.

The author is with the Hong Kong Baptist University, Kowloon Tong, Hong Kong (e-mail: prof_sim_2002@yahoo.com).

Color versions of one or more of the figures in this paper are available online at http://ieeexplore.iee.org.

Digital Object Identifier 10.1109/TSMCB.2008.928210 gotiation pressure. In some applications (e.g., grid resource management [2]-[6]), being more successful in negotiating for resources more rapidly is a desirable property because any delay incurring on waiting for resources would be perceived as an overhead [2]. Sim and Wang's [21] preliminary work on relaxed-criteria negotiation enhanced the flexibility of marketdriven agents (MDAs) [18]-[20] (see Section II) by augmenting them with sets of fuzzy rules that guide these agents in relaxing their bargaining terms (e.g., proposed trading price) in the face of (intense) negotiation pressure (e.g., very stiff competition). Preliminary results in [5], [6], and [21] have shown that designing negotiation agents with the flexibility to relax their bargaining terms enhanced their chances of successfully reaching agreements and perhaps reaching agreements more rapidly. Nevertheless, even though the designs of negotiation agents in [5], [6], and [21] were augmented with fuzzy controllers and fuzzy rules, the fuzzy rules in [5], [6], and [21] were manually constructed, and the system structures of these negotiation agents remained generally fixed throughout their operations in different e-markets. Since there are many varying parameters in an e-market, e.g., there can be different numbers of participants, different numbers of buyers and sellers, and different constraints such as different negotiation deadlines, it is difficult to find a fixed set of fuzzy rules that is suitable for all e-markets. The impetus of this work is designing and developing negotiation agents that not only have the flexibility of relaxing the bargaining criteria using fuzzy rules, but also has the ability to evolve their structures by learning new relaxedcriteria fuzzy rules to improve their negotiation outcomes as they participate in negotiations in more e-markets with unpredictable market conditions (e.g., agents can enter/leave an e-market at any time).

The novel features of this work in relaxed-criteria are:

1) formulating a new relaxed-criteria negotiation model (Section III);

2) specifying an entirely new set of criteria for negotiation agents to relax their bargaining terms (Section III-B);

3) designing a new fuzzy decision controller (FDC) (Section III-C) for agents to determine the amount of relaxation in a negotiation situation by consulting a set of relaxed-criteria fuzzy rules;

4) designing an evolutionary algorithm (Section IV-A) that invokes a genetic algorithm (GA) (Section IV-B) for evolving and adapting the relaxed-criteria rules as the negotiation agents participate in negotiation activities in a series of e-markets;

5) designing and implementing a testbed and conduct experimentations (Section $\mathrm{V}$ ) using the testbed to evaluate 
the effectiveness of the evolutionary algorithm in 4) for evolving relaxed-criteria fuzzy rules.

Section VI compares this paper with existing negotiation agents that are designed with the flexibility to relax their trading conditions using fuzzy approaches. Section VII concludes this paper by summarizing a list of contributions and future works.

\section{Market-Driven Negotiation Agent}

An MDA is an e-negotiation agent that determines the appropriate amounts of concessions using three negotiation decision functions: time $(T)$, competition $(C)$, and opportunity $(O)$ [18]-[20]. It makes concessions by narrowing the difference $k_{t}$ between its proposal in a negotiation round $t$ and its reserve price (RP). In determining the appropriate amounts of concessions, Sim has shown in [18] and [19] that an MDA should adopt the opportunity, competition, and time decision functions to determine the expected difference $k_{t+1}$ between its proposal in round $t+1$ and its $\mathrm{RP}$ :

$$
\begin{aligned}
k_{t+1}=f\left[\boldsymbol{O}\left(n_{t}^{B}, v_{t}^{B \rightarrow S j},\left\langle w_{t}^{S j \rightarrow B}\right\rangle\right),\right. & \\
& \left.\boldsymbol{C}\left(m_{t}^{B}, n_{t}^{B}\right), \boldsymbol{T}(t, \tau, \lambda)\right] k_{t} .
\end{aligned}
$$

Since an MDA makes concession by narrowing $k_{t}$ in the next round, $k_{t+1}$ is a fraction of $k_{t}$. Let $P_{t}$ be the proposal of an MDA at round $t$. An MDA that is a seller generates its proposal in round $t+1$ by decrementing its price as follows:

$$
\begin{array}{r}
P_{t+1}=P_{t}-\left(k_{t}-k_{t+1}\right) \\
=P t-\left(1-f\left[\boldsymbol{O}\left(n_{t}^{B}, v_{t}^{B \rightarrow S j},\left\langle w_{t}^{S j \rightarrow B}\right\rangle\right),\right.\right. \\
\left.\left.\boldsymbol{C}\left(m_{t}^{B}, n_{t}^{B}\right), \boldsymbol{T}(t, \tau, \lambda)\right]\right) k_{t}
\end{array}
$$

where $k_{t}=P_{t}-\mathrm{RP}$.

An MDA that is a buyer generates its proposal in round $t+1$ by incrementing its price as follows:

$$
\begin{array}{r}
P_{t+1}=P_{t}+\left(k_{t}-k_{t+1}\right) \\
=P_{t}+\left(1-f\left[\boldsymbol{O}\left(n_{t}^{B}, v_{t}^{B \rightarrow S j},\left\langle w_{t}^{S j \rightarrow B}\right\rangle\right),\right.\right. \\
\left.\left.\boldsymbol{C}\left(m_{t}^{B}, n_{t}^{B}\right), \boldsymbol{T}(t, \tau, \lambda)\right]\right) k_{t}
\end{array}
$$

where $k_{t}=\mathrm{RP}-P_{t}$.

1) Opportunity Function: The opportunity function $\boldsymbol{O}\left(n_{t}^{B}\right.$, $\left.v_{t}^{B \rightarrow S j},\left\langle w_{t}^{S j \rightarrow B}\right\rangle\right)=1-\prod_{j=1}^{n_{t}^{B}}\left(\left(v_{t}^{B \rightarrow S j}-w_{t}^{S j \rightarrow B}\right) /\left(v_{t}^{B \rightarrow S j}-c^{B}\right)\right)$ determines the amount of concession based on 1) trading alternatives (i.e., outside options or number of trading parties $n_{t}^{B}$ ) and 2) differences in utilities generated by the proposal of an MDA $\left(v_{t}^{B \rightarrow S j}\right)$ and the counterproposal(s) of its trading party(parties) $\quad\left(\left\langle w_{t}^{S j \rightarrow B}\right\rangle=\left\{w_{t}^{S 1 \rightarrow B}, w_{t}^{S 2 \rightarrow B}, \ldots, w_{t}^{S j \rightarrow B}\right\}\right)$ [18]-[20]. $c^{B}$ is the worst possible utility for agent $B$. When negotiation ends in a conflict (i.e., $B$ fails to reach an agreement with its trading parties), $B$ obtains its worst outcome $c^{B}$. Whereas $v_{t}^{B \rightarrow S j}-w_{t}^{S j \rightarrow B}$ measures the cost of accepting a trading party's $\left(S_{j}^{\prime} s\right)$ last offer (i.e., difference between the (counter-)proposals of $B$ and $S_{j}$ ), $v_{t}^{B \rightarrow S j}-c^{B}$ measures the cost of provoking a conflict. When determining opportunity, it was shown in [18] and [19] that if there is a large number of trading alternatives, the likelihood that an agent proposes a bid/offer that is potentially close to an MDA's offer/bid may be high. However, it would be difficult for the MDA to reach a consensus if none of the so many options are viable (i.e., there are large differences between the proposal of an MDA and the counterproposals of all its trading parties). On this account, the $\boldsymbol{O}$ function determines the probability of reaching a consensus on its own terms by determining its bargaining position based on 1) trading alternatives and 2) differences between its proposal and proposals of other agents. The general idea is that if the probability of reaching a consensus on its own terms is high (respectively, low), an MDA will make a smaller (respectively, larger) amount of concession.

2) Competition Function: $\boldsymbol{C}\left(m_{t}^{B}, n_{t}^{B}\right)$ determines the amount of competition of an MDA by determining the probability that it is not being considered as the most preferred trading party. Since MDAs are utility maximizing agents, an MDA is more likely to reach a consensus if its proposal is ranked the highest by some other agent. Suppose an agent $B_{1}$ has $m_{t}^{B}-1$ competitors $\left\{B_{2}, \ldots, B_{m}\right\}$ and $n_{t}^{B}$ trading parties $\left\{S_{1}, \ldots, S_{n}\right\}$. The probability that $B_{1}$ is not the most preferred trading party of any $S_{j}$ (where $S_{j} \in\left\{S_{1}, \ldots, S_{n}\right\}$ ) is $\left(m_{t}^{B}-1\right) / m_{t}^{B}$. Hence, the probability that $B_{1}$ is not the most preferred party of all $S_{j} \in\left\{S_{1}, \ldots, S_{n}\right\}$ is $\left[\left(m_{t}^{B}-1\right) / m_{t}^{B}\right]^{n_{t}^{B}}$. Hence, the probability that $B_{1}$ is considered the most preferred trading party by at least one of $S_{j} \in\left\{S_{1}, \ldots, S_{n}\right\}$ is as follows: $C\left(m_{t}^{B}, n_{t}^{B}\right)=1-\left[\left(m_{t}^{B}-1\right) / m_{t}^{B}\right]^{n_{t}^{B}}$, where $m_{t}^{B}$ and $n_{t}^{B}$ are the numbers of buyer agents (including $B_{1}$ ) and seller agents at round $t$, respectively. The general ideas of $\boldsymbol{C}\left(m_{t}^{B}, n_{t}^{B}\right)$ are the following: 1) the probability of being considered the most preferred trading party by some parties increases with the number of trading parties $n_{t}^{B}$ and 2) with a larger number of competitors $m_{t}^{B}$, the likelihood of being considered the most preferred trading party decreases. If the probability of being the most preferred trading party is high (respectively, low), an MDA will make a smaller (respectively, larger) amount of concession.

3) Time Function: The time-dependent function $T(t, \tau, \lambda)$ models the intuition that as time passes, an MDA relaxes its proposal by attempting to narrow $k_{t}$ using: $T(t, \tau, \lambda)=1-$ $(t / \tau)^{\lambda}$, where $t$ is the current trading time, $\tau$ is the deadline, and $\lambda$ is an MDA's time preference. Negotiators with different time preferences may adopt different concession rates with respect to time. The concession rate is determined with respect to $0<$ $\lambda<\infty$. With infinitely many values of $\lambda$, there are infinitely many possible strategies in making concession with respect to remaining trading time. However, they are classified in $[18]-[20]$ as follows.

1) Linear: $\lambda=1$ and $P_{t+1}=P_{t}-[1-T(t, \tau, \lambda)] k_{t}=P_{t}-$ $(t / \tau) k_{t}$ (respectively, $P_{t+1}=P_{t}+[1-T(t, \tau, \lambda)] k_{t}=$ $\left.P_{t}+(t / \tau) k_{t}\right)$ for seller (respectively, buyer). At any round $t$, an MDA makes a constant rate of concession, $\Delta_{t}=\left|P_{t}-P_{t+1}\right|=(t / \tau) k_{t}$. At the deadline $t=$ $\tau, \Delta_{t}=(\tau / \tau) k_{t}=k_{t}$ (an MDA attempts to narrow the difference completely at the deadline), and for a seller (respectively, buyer) $P_{t+1}=P_{t}-(\tau / \tau) k_{t}=\mathrm{RP}$ (respectively, $\left.P_{t+1}=P_{t}+(\tau / \tau) k_{t}=\mathrm{RP}\right) \quad$ (an MDA proposes RP at its deadline). 
2) Conciliatory: $0<\lambda<1$ and $P_{t+1}=P_{t}-(t / \tau)^{\lambda} k_{t}$ (respectively, $P_{t+1}=P_{t}+(t / \tau)^{\lambda} k_{t}$ ) for seller (respectively, buyer). An MDA makes larger concessions in the early trading rounds and smaller concessions at the later stage.

3) Conservative: $1<\lambda<\propto$ and $P_{t+1}=P_{t}-(t / \tau)^{\lambda} k_{t}$ (respectively, $P_{t+1}=P_{t}+(t / \tau)^{\lambda} k_{t}$ ) for seller (respectively, buyer). An MDA makes smaller concessions in early rounds and larger concessions in later rounds.

\section{RELAXED-CRITERIA NeGOTIATION MODEL}

In the alternating offers protocol and also in most negotiation models (e.g., [7]-[17], only to name a few because of space limitation), a pair of negotiation agents $\left(B_{1}, S_{1}\right)$ reaches an agreement when one agent proposes a deal that matches (or exceeds) what another agent asks for. The negotiation protocol in [3], [5], [6], and [21] enhances the alternating offers protocol [10] by slightly relaxing the criteria for agents to reach a consensus. The relaxed-criteria negotiation protocol in this work is given in Section III-A. Relaxation of negotiation criteria (Section III-B) is achieved by augmenting negotiation agents with an FDC (Section III-C).

\section{A. Relaxed-Criteria Negotiation Protocol}

Following [3], [5], [6], and [21], the negotiation activities among buyer agents and seller agents are specified as follows.

1) Negotiation proceeds in a series of rounds.

2) Adopting Rubinstein's alternating offers protocol [10, p. 100], a pair of buyer and seller agents negotiates by making proposals in alternate rounds.

3) Multiple buyer-seller agent pairs can negotiate deals simultaneously.

4) When an agent makes a proposal, it proposes a deal from its space of possible deals (e.g., consisting of the most desirable price, the least desirable (reserve) price, and those prices in between). Typically, an agent proposes its most preferred deal initially [23].

5) If no agreement is reached, negotiation proceeds to the next round. At every round, an agent determines its amount of concession using the $\boldsymbol{T}, \boldsymbol{O}$, and $\boldsymbol{C}$ functions [18]-[20] (see Section II).

6) Negotiation between two agents terminates 1) when an agreement is reached or 2) with a conflict when one of the bargaining agents' deadline is reached.

7) Agents follow one of the following rules for reaching an agreement.

R1) An agreement is reached if an agent $B_{1}$ and its trading party $S_{1}$ propose deals $b_{1}$ and $o_{1}$, respectively, such that either 1) $U\left(b_{1}\right) \geq U\left(o_{1}\right)$ or 2) $U\left(o_{1}\right) \geq U\left(b_{1}\right)$, where $b_{1}$ and $o_{1}$ represent the buying and selling prices and $U$ is a utility function.

R2) An agreement is reached if either 1) $\eta=U\left(o_{1}\right)-U\left(b_{1}\right)$, such that $\eta \rightarrow 0$ or 2) $\eta=U\left(b_{1}\right)-U\left(o_{1}\right)$, such that $\eta \rightarrow 0$, where $\eta$ is the amount of relaxation determined using an FDC described in Section III-C (details can be found in [5], [6], and [21]).

\section{B. Relaxation Criteria}

Whereas, agents in [21] use eagerness and degree of competition to determine the amounts of relaxation of the negotiation conditions, this work considers three relaxation criteria: 1) degree of competition $(\vartheta)$; 2) time pressure $\left(T_{r}\right)$; and 3$)$ the relative distances from trading parties' proposals $(\delta)$.

1) Degree of Competition: $\vartheta \in[0,1]$ represents the degree of competition that an agent faces at round $t$. An agent determines its degree of competition at round $t$ by considering the number of its competitors and the number of its trading parties as follows:

$$
\vartheta=\left[\left(m_{t}^{a}-1\right) / m_{t}^{a}\right]^{n_{t}^{a}}
$$

where $m_{t}^{a}-1$ is the number of the agents' competitors at round $t$, and $n_{t}^{a}$ is the number of the agent's trading parties at round $t$. With a higher (respectively, lower) value of $\vartheta$, an agent faces more (respectively, less) competition, and is more (respectively, less) likely to relax its bargaining criteria to reach an agreement.

2) Time Pressure: Let $T_{r} \in[0,1]$ represents an agent's time pressure. An agent determines its time pressure as follows. $T_{r}=t / \tau$, where $\tau$ is the agent's deadline and $t$ is the time index. When an agent's deadline is fast approaching (i.e., $T_{r} \rightarrow 1$ ), it is under more pressure to relax its bargaining criteria.

3) Distances From Trading Parties' Proposals: Another criterion for relaxation is the relative distances between the proposal of an agent and all the proposals of its trading parties. The general idea is that if the best proposal from an agent's trading party is very close to its own proposal relative to all other proposals from all other trading parties, then it seems prudent that an agent should relax its bargaining terms and reach a consensus with the trading party with the best proposal. Let $\delta \in[0,1]$ represents relative distances between the proposal of an agent and all the proposals of its trading parties. At each round $t$, an agent determines $\delta$ as follows:

$$
\delta\left(d_{t}^{\mathrm{best}}, d_{t}^{i}\right)=1-\frac{d_{t}^{\mathrm{best}}}{\left(\sum_{i}^{n_{t}^{a}} d_{t}^{i}-d_{t}^{\mathrm{best}}\right) /\left(n_{t}^{a}-1\right)}
$$

where $d_{t}^{i}$ denotes the distance between an agent's proposal and its $i$ th trading party's proposal at round $t, d_{t}^{\text {best }}=$ $\min \left(d_{t}^{1}, d_{t}^{2}, \ldots, d_{t}^{n_{t}^{a}}\right)$, and $n_{t}^{a}$ is the number of the agent's trading parties at round $t$. A high value of $\delta$ indicates that the best proposal from its trading parties is very close to an agent's proposal relative to all other proposals from all other trading parties.

Factors such as degree of competition, time pressure, and distances from trading parties generally place an agent under pressure. When an agent is under (intense) pressure (e.g., when it is facing stiff competition, or when its deadline is fast approaching, or when its own proposal is relatively close to a trading party's proposal and relatively far from all other proposals of its trading parties, it is more likely to relax its negotiation criteria to enhance its probability of successfully reaching agreements and perhaps reaching agreements more 
TABLE I

Manually Designed FuZzy Rules

\begin{tabular}{|c|c|c|c|c|}
\hline No & If $\vartheta$ & And $T_{r}$ & And $\delta$ & Then $\eta$ \\
\hline 1 & $\mathrm{~L}$ & $\mathrm{~L}$ & $\mathrm{~L}$ & $\mathrm{~L}$ \\
\hline 2 & $\mathrm{~L}$ & L & $\mathrm{M}$ & L \\
\hline 3 & $\mathrm{~L}$ & $\mathrm{~L}$ & $\mathrm{H}$ & $\mathrm{M}$ \\
\hline 4 & $\mathrm{~L}$ & M & $\mathrm{L}$ & L \\
\hline 5 & $\mathrm{~L}$ & M & $\mathrm{M}$ & M \\
\hline 6 & $\mathrm{~L}$ & M & $\mathrm{H}$ & $\mathrm{M}$ \\
\hline 7 & $\mathrm{~L}$ & $\mathrm{H}$ & $\mathrm{L}$ & $\mathrm{M}$ \\
\hline 8 & $\mathrm{~L}$ & $\mathrm{H}$ & $\mathrm{M}$ & $\mathrm{M}$ \\
\hline 9 & $\mathrm{~L}$ & $\mathrm{H}$ & $\mathrm{H}$ & $\mathrm{M}$ \\
\hline 10 & M & $\mathrm{L}$ & $\mathrm{L}$ & $\mathrm{L}$ \\
\hline 11 & M & $\mathrm{L}$ & M & $\mathrm{L}$ \\
\hline 12 & M & L & $\mathrm{H}$ & $\mathrm{M}$ \\
\hline 13 & M & M & L & L \\
\hline 14 & M & M & M & $\mathrm{M}$ \\
\hline 15 & M & $\mathrm{M}$ & $\mathrm{H}$ & $\mathrm{H}$ \\
\hline 16 & M & $\mathrm{H}$ & $\mathrm{L}$ & M \\
\hline 17 & M & $\mathrm{H}$ & M & $\mathrm{H}$ \\
\hline 18 & M & $\mathrm{H}$ & $\mathrm{H}$ & $\mathrm{H}$ \\
\hline 19 & $\mathrm{H}$ & $\mathrm{L}$ & L & L \\
\hline 20 & $\mathrm{H}$ & $\mathrm{L}$ & $\mathrm{M}$ & $\mathrm{L}$ \\
\hline 21 & $\mathrm{H}$ & $\mathrm{L}$ & $\mathrm{H}$ & M \\
\hline 22 & $\mathrm{H}$ & M & $\mathrm{L}$ & L \\
\hline 23 & $\mathrm{H}$ & M & $\mathrm{M}$ & $\mathrm{M}$ \\
\hline 24 & $\mathrm{H}$ & M & $\mathrm{H}$ & $\mathrm{H}$ \\
\hline 25 & $\mathrm{H}$ & $\mathrm{H}$ & $\mathrm{L}$ & M \\
\hline 26 & $\mathrm{H}$ & $\mathrm{H}$ & M & $\mathrm{M}$ \\
\hline 27 & $\mathrm{H}$ & $\mathrm{H}$ & $\mathrm{H}$ & $\mathrm{H}$ \\
\hline
\end{tabular}

rapidly. Since notions such as "stiff" competition, and "fast" approaching deadline, and relatively "close" to or "far" from a trading party' proposal are vague, it is prudent to use fuzzy sets to represent these concepts, and to design an FDC for guiding agents' decisions for relaxing the negotiation conditions.

\section{Fuzzy Decision Controller}

The FDC has three inputs $\left(\vartheta, T_{r}\right.$, and $\left.\delta\right)$ and one output $\eta$ (degree of relaxation), and it consults a set of fuzzy rules (see Table I for an example of a set of relaxed-criteria fuzzy rules). It consists of a fuzzification interface (FI), a fuzzy rule base (RB), a fuzzy negotiation decision-making logic (DML), and a defuzzification interface (DFI).

1) Fuzzification: The FI converts the (crisp) input data to the FDC $\left(\vartheta, T_{r}\right.$, and $\left.\delta\right)$ into linguistic values (or labels of fuzzy sets). Based on the range of possible values of a variable, several fuzzy sets (i.e., $L_{\vartheta}, M_{\vartheta}, H_{\vartheta} ; L_{T_{r}}, M_{T_{r}}, H_{T_{r}}$; and $L_{\delta}$, $\left.M_{\delta}, H_{\delta}\right)$ are defined. That is, each variable has three fuzzy values: $L, M$, and $H$. For example, for $\vartheta$, the fuzzy set $L_{\vartheta}$ corresponds to the range between $\vartheta=0$ and $\vartheta=1 / 2$. Some examples of linguistic values of the variables $\vartheta, T_{r}$, and $\delta$ are "stiff competition" (represented as $H$ in Table I), "high time pressure," and "relatively close to a trading party's proposal," respectively. A membership function $\mu(x)$ is used for assigning the membership degree of each crisp value of a variable in the fuzzy sets [24]. The following membership functions are used to assign the degree of membership for $\vartheta, T_{r}, \delta$, and $\eta$ :

$$
\mu(x)=\left\{\begin{array}{l}
-2 x+1, x \in[0,1 / 2] \\
p_{1}(2 x)+\left(1-p_{1}\right)(-2 x+1), x \in[0,1] \\
2 x-1, x \in[1 / 2,1]
\end{array}\right.
$$

where $p_{1}=1$ when $x \in[0,1 / 2]$, and $p_{1}=0$ when $x \in$ $[1 / 2,1]$. For example, a crisp value $\vartheta=0.2$ may be "fuzzified" as a member of $L$ with a membership degree of 0.6 , and as a member of $M$ with a membership degree of 0.4 (see Fig. 1).

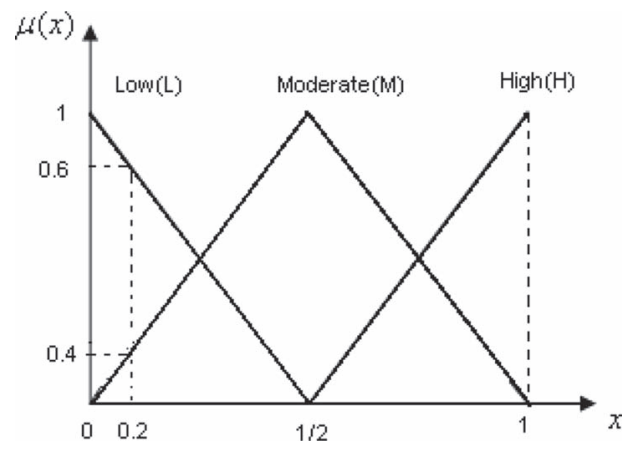

Fig. 1. Linguistic terms of the membership function.

2) Fuzzy Decision Making: By consulting the fuzzy rules (see Table I) in RB, the DML infers the linguistic value of $\eta$ and its corresponding membership degree $\mu(\eta)$ from the linguistic values and membership degrees of the fuzzified inputs $\vartheta, T_{r}$, and $\delta$. For example, suppose that $\vartheta$ is a member of $M$ with $\mu(\vartheta)=0.38$ and a member of $H$ with $\mu(\vartheta)=0.62$, and $T_{r}$ is a member of $L$ with $\mu\left(T_{r}\right)=0.88$ and a member of $M$ with $\mu\left(T_{r}\right)=0.12$, and $\delta$ is a member of $M$ with $\mu(\delta)=0.44$ and a member of $H$ with $\mu(\delta)=0.56$. Guided by rules 11, 12, 14, 15, 20, 21, 23, and 24 in Table I (chosen based on the combination of the linguistic values of $\vartheta, T_{r}$, and $\delta$ ), the DML infers that $\eta$ is a member of $L$ with $\mu(\eta)=0.39$ and a member of $M$ with $\mu(\eta)=0.54$ and a member of $\mathrm{H}$ with $\mu(\eta)=0.07$. The membership of $\eta$ for a linguistic value is determined by $\mu(\eta)=\sum \mu(\vartheta) \times \mu\left(T_{r}\right) \times \mu(\delta)$. For instance, by rule 11 , if $\vartheta$ is a member of $M$ with $\mu(\vartheta)=0.38$, and $T_{r}$ is a member of $L$ with $\mu\left(T_{r}\right)=0.88$, and $\delta$ is a member of $M$ with $\mu(\delta)=0.44$, then $\eta$ is a member of $L$ with $\mu(\eta)=\mu(\vartheta) \times \mu\left(T_{r}\right) \times \mu(\delta)=0.38 \times 0.88 \times 0.44=0.15$. Similarly, by rule $12, \eta$ is a member of $M$ with $\mu(\eta)=\mu(\vartheta) \times \mu\left(T_{r}\right) \times \mu(\delta)=0.38 \times 0.88 \times 0.56=0.19$. By rule $14, \eta$ is a member of $M$ with $\mu(\eta)=$ $\mu(\vartheta) \times \mu\left(T_{r}\right) \times \mu(\delta)=0.38 \times 0.12 \times 0.44=0.02$. By rule $15, \eta$ is a member of $H$ with $\mu(\eta)=\mu(\vartheta) \times \mu\left(T_{r}\right) \times \mu(\delta)=$ $0.38 \times 0.12 \times 0.56=0.03$. By rule $20, \eta$ is a member of $L$ with $\mu(\eta)=\mu(\vartheta) \times \mu\left(T_{r}\right) \times \mu(\delta)=0.62 \times 0.88 \times$ $0.44=0.24$. By rule $21, \eta$ is a member of $M$ with $\mu(\eta)=\mu(\vartheta) \times \mu\left(T_{r}\right) \times \mu(\delta)=0.62 \times 0.88 \times 0.56=0.30$. By rule 23, $\eta$ is a member of $M$ with $\mu(\eta)=$ $0.62 \times 0.12 \times 0.44=0.03$. By rule $24, \eta$ is a member of $H$ with $\mu(\eta)=0.62 \times 0.12 \times 0.56=0.04$. Hence, by rules 11 and $20, \eta$ is a member of $L$ with $\mu(\eta)=0.15+0.24=0.39$. By rules $12,14,21$, and $23, \eta$ is a member of $M$ with $\mu(\eta)=0.19+0.02+0.30+0.03=0.54$. By rules 15 and 24, $\eta$ is a member of $H$ with $\mu(\eta)=0.03+0.04=0.07$.

3) Defuzzification: The DFI is used to determine the crisp value of $\eta$ given its linguistic values with their respective membership degrees being obtained from the DML. To compute the crisp value of $\eta$, the DFI in this work adopts the weighted average method [25]. For example, given that $\eta$ is a member of $L$ with $\mu(\eta)=0.8$ and a member of $M$ with $\mu(\eta)=0.2$ which are obtained from the DML, DFI is used to determine a single crisp value of $\eta$ as follows:

$$
\eta=(0.8 \times 0+0.2 \times(1 / 2)) /(0.8+0.2)=0.1 .
$$


1. Manually design a fuzzy rule set $R_{0}$ containing 27 fuzzy rules.

2. Incorporate $R_{0}$ into the FDCs of MDAs and simulate the negotiation process of $M D A s$ in e-market $M_{0}$.

3. Record the crisp values of $\vartheta, T_{r}, \delta$, and $\eta$ of $M D A s$ that have reached agreements as a data set $D_{0}$.

4. Set the iteration count $i$ to 0 .

5. While not stop,

a. Invoke procedure $\mathbf{G A}\left(R_{i}, D_{i}\right)$ to evolve a set of new fuzzy rules.

b. Replace the corresponding rules in $R_{i}$ with the newly evolved fuzzy rules, and copy all the rules in $R_{i}$ to a new fuzzy rule set $R_{i+1}$.

c. Incorporate $R_{i+1}$ into the FDCs of MDAs and simulate the negotiation process of MDAs in e-market $M_{\mathrm{i}+1}$.

d. Record the crisp values of $\vartheta, T_{r}, \delta$, and $\eta$ of $M D A s$ that have reached agreements as a data set $D_{i+1}$.

e. Increment $i$ by 1 .

Fig. 2. Procedure for evolving relaxed-criteria rules.

Consequently, the crisp value of $\eta$ is used to determine if an agent's trading party's proposal is sufficiently close to its own, so that an agreement can be reached following the relaxedcriteria protocol described in Section III-A.

\section{Evolving Relaxed-CRITERIA RULES}

Whereas negotiation agents described in [3], [5], [6], and [21] are designed to reach agreements with more flexibility, the relaxed-criteria rules are manually constructed. This section describes an approach in which relaxed-criteria rules of MDAs are evolved as they negotiate in a series of different emarkets. In this work, an evolutionary procedure (Section IV-A) is proposed for learning effective relaxed-criteria negotiation rules that would enhance the performance of MDAs in terms of success rates in negotiation, expected utility, negotiation speed, and combined negotiation outcomes. Using the negotiation outcomes of agents in each e-market as data sets, at the termination of the negotiation process for each e-market, a GA (Section IV-B) is executed to evolve a new set of (fuzzy) relaxed-criteria rules of MDAs.

\section{A. Algorithm for Evolving Fuzzy Rules}

An evolutionary procedure (see Fig. 2) is designed to improve the negotiation performance of MDAs by evolving their relaxed-criteria (fuzzy) rule sets as they negotiate in a series of different e-markets $\left\{M_{0}, M_{1}, \ldots\right\}$. Initially, a set $R_{0}$ of 27 manually designed fuzzy rules is generated (see Table I). During the negotiation process in e-market $M_{0}$, MDAs adopt $R_{0}$ as their relaxed-criteria rule sets in their FDCs for determining if agreements should be reached. When the negotiation process of $M_{0}$ terminates, the crisp values of $\vartheta, T_{r}, \delta$, and $\eta$ of MDAs that have successfully reached agreements are recorded as a data set $D_{0}$. Subsequently, using both $D_{0}$ and $R_{0}$ as inputs, the GA in Section IV-B is invoked to evolve a set of new fuzzy rules. The set of newly evolved fuzzy rules replaces some of the rules

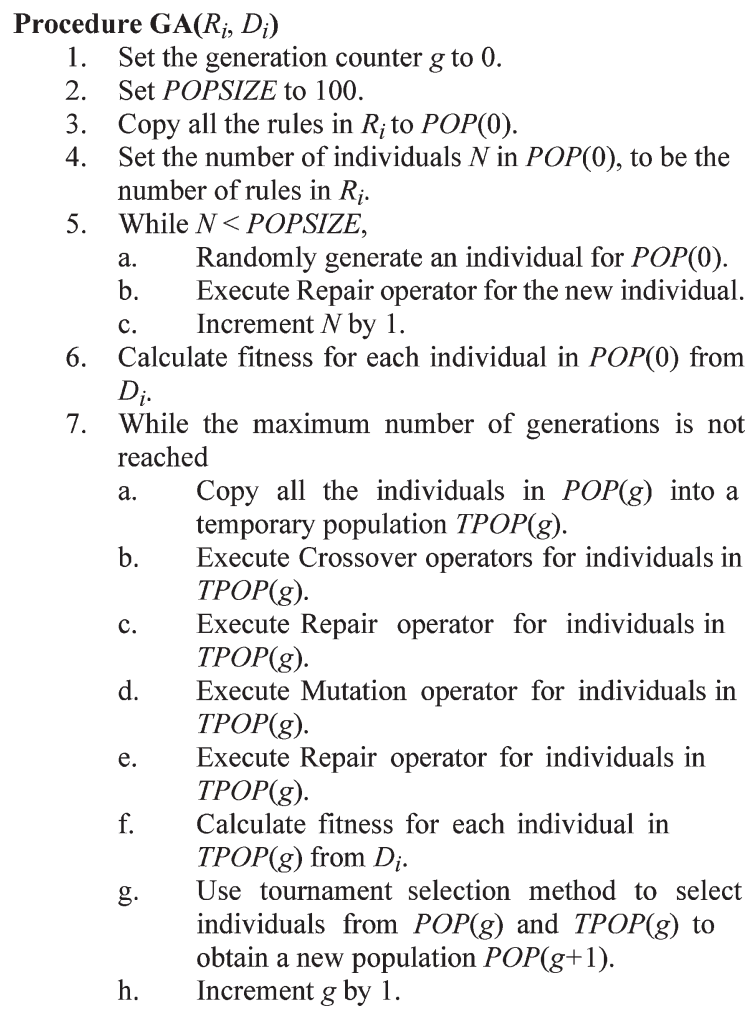

6. Calculate fitness for each individual in $P O P(0)$ from $D_{i}$.

7. While the maximum number of generations is not reached

a. Copy all the individuals in $P O P(g)$ into a temporary population $T P O P(g)$.

b. Execute Crossover operators for individuals in TPOP $(g)$.

c. Execute Repair operator for individuals in $T P O P(g)$.

d. Execute Mutation operator for individuals in $T P O P(g)$.

e. Execute Repair operator for individuals in $T P O P(g)$.

f. Calculate fitness for each individual in $\operatorname{TPOP}(g)$ from $D_{i}$.

g. Use tournament selection method to select individuals from $P O P(g)$ and $T P O P(g)$ to obtain a new population $P O P(g+1)$.

h. Increment $g$ by 1 .

8. Decode the best individual into relaxed-criteria rules, and return the rules.

Fig. 3. GA for evolving relaxed-criteria rules.

in $R_{0}$ to form a new rule set $R_{1}$. The process continues such that at each $\left.M_{i}: 1\right)$ MDAs adopt $R_{i}$ as their relaxed-criteria rule sets; 2 ) when the negotiation process of $M_{i}$ terminates, the crisp values of $\vartheta, T_{r}, \delta$, and $\eta$ of MDAs that have successfully reached agreements are recorded as a data set $D_{i}$; and 3) using both $D_{i}$ and $R_{i}$ as inputs, the GA in Section IV-B is invoked to evolve a new set of fuzzy rules which replace some of the rules in $R_{i}$ to form a new rule set $R_{i+1}$ which will be adopted by MDAs in $M_{i+1}$.

\section{B. Genetic Algorithm}

This section describes the GA (see Fig. 3) used for evolving relaxed-criteria (fuzzy) rules, including the encoding and decoding methods, the fitness function, and the genetic operators. Accepting two inputs: 1) a rule set $R_{i}$ and 2) a data set $D_{i}$, the GA outputs a set of newly evolved relaxed-criteria rules. Whereas, $R_{i}$ is used for initializing the population in the GA, $D_{i}$ is used for calculating the fitness of each individual. The details are illustrated as follows.

1) Encoding Relaxed-Criteria Rules as Genes: In this work, relaxed-criteria rules are encoded as strings with fixed length (individuals). Each string, which consists of " $m_{1} m_{2} m_{3} m_{4}$ " is an abstract representation of a fuzzy rule (relaxed-criteria rule), where $m_{1}, m_{2}$, and $m_{3}$ represent the antecedents and $m_{4}$ represents the conclusion of a fuzzy rule. The value domain for each variable in the antecedent and conclusion of a fuzzy rule is $\{0,1,2,3\}$. Whereas " 1, ," 2 ," and " 3 " are used to represent the fuzzy values " $L$," “ $M$," and " $H$," respectively, " 0 " represents 
that the corresponding variable does not appear in the fuzzy rule. Hence, each string may encode several different rules. For example, string "1232" represents the rule "IF $\vartheta$ is $L$ AND $T_{r}$ is $M$ AND $\delta$ is $H$, THEN $\eta$ is $M$," string " 0111 " represents "IF $T_{r}$ is $L$ AND $\delta$ is $L$, THEN $\eta$ is $L$," regardless of the value of $\vartheta$. Thus, string " 0111 " potentially encodes three different rules.

2) Decoding Genes to Relaxed-Criteria Rules: Each string can be decoded to one or several relaxed-criteria rules containing three variables in the antecedent and one variable in the conclusion. Since " 0 " in a string represents that the corresponding variable does not appear in a fuzzy rule, the variable can be mapped into any value in the domain. For example, whereas string " 0121 " can be translated into three different rules corresponding to "1121," "2121," and "3121," string "0101"can be translated into nine different rules.

3) Fitness Function: In this paper, the fitness of each individual $m$ with a string " $m_{1} m_{2} m_{3} m_{4}$ " is determined as follows:

$$
\text { fitness }(m)=\frac{\mathrm{TP}}{\mathrm{TP}+\mathrm{FN}} \times \frac{\mathrm{TN}}{\mathrm{FP}+\mathrm{TN}} .
$$

The symbols are described as follows. A data record is positive $(P)$ if it is covered by $m$, and it is negative $(N)$ if it is not covered by $m$. If a data record is predicted to be $P$ (i.e., the antecedent is covered by $m$, and it is predicted that $m$ also covers the conclusion) and the outcome is actually $P$ (i.e., the conclusion is actually covered by $m$ ), then it is called a true positive [26]. However, if the conclusion is actually $N$ (i.e., the conclusion is actually not covered by $m$ ), then it is called a false positive. Conversely, a true negative occurs when a data record is predicted to be $N$ (i.e., the antecedent is not covered by $m$, and it is predicted that the conclusion is also not covered by $m$ ) and the outcome is actually $N$ (i.e., the actual conclusion is not covered by $m$ ). A false negative occurs when a data record is predicted to be $N$ but the outcome is actually $P$. Hence, TP (true positives) [26] is the number of data records that are covered by the antecedent and conclusion of $m$. FP (false positives) [26] is the number of data records that are covered by the antecedent but not the conclusion of $m$. TN (true negatives) [26] is the number of records that are not covered by both the antecedent and conclusion of $m$. FN (false negatives) [26] is the number of records that are covered by $m$ 's conclusion but not its antecedent.

In this work, a data record $d$ is covered by individual $m$ if it satisfies the following.

CR1) If $\min \left\{\mu_{m_{1}}\left(d_{1}\right), \mu_{m_{2}}\left(d_{2}\right), \mu_{m_{3}}\left(d_{3}\right)\right\} \geq \varepsilon$, then it is considered that data record $d$ is covered by the antecedent of individual $m$ (if $m_{i}=0$, then $\mu_{m_{i}}\left(d_{i}\right)$ is ignored and deleted from the formula $\min \left\{\mu_{m_{1}}\left(d_{1}\right)\right.$, $\left.\left.\mu_{m_{2}}\left(d_{2}\right), \mu_{m_{3}}\left(d_{3}\right)\right\}, i=1,2,3\right)$;

CR2) If $\mu_{m_{4}}\left(d_{4}\right) \geq \varepsilon$, then it is considered that data record $d$ is covered by the conclusion of individual $m$.

$d_{1}, d_{2}, d_{3}$, and $d_{4}$ represent $\vartheta, T_{r}, \delta$, and $\eta$, respectively, $\mu_{m_{i}}\left(d_{i}\right)$ denotes the membership degree of the $i$ th variable if its crisp value is $d_{i}$ and its linguistic value is represented by $m_{i}(i=1,2,3,4)$, and $\varepsilon$ is set to be 0.5 . For instance, suppose that data record $d=\{0.2,0.4,0.1,0.05\}$ and individual $m=$ "1211". String "1211" represents the rule "IF $\vartheta$ is $L$ AND
$T_{r}$ is $M$ AND $\delta$ is $L$, THEN $\eta$ is $L . "$ Then, $\mu_{m_{1}}\left(d_{1}\right)=$ $\mu_{1}(0.2)=\mu_{L}(0.2)=0.6, \quad \mu_{m_{2}}\left(d_{2}\right)=\mu_{2}(0.4)=\mu_{M}(0.2)=0.8$, $\mu_{m_{3}}\left(d_{3}\right)=\mu_{1}(0.1)=0.8$, and $\mu_{m_{4}}\left(d_{4}\right)=\mu_{1}(0.05)=0.9$. According to (CR1), $\min \left\{\mu_{m_{1}}\left(d_{1}\right), \mu_{m_{2}}\left(d_{2}\right), \mu_{m_{3}}\left(d_{3}\right)\right\}=$ $0.6>\varepsilon$, hence, $d$ is covered by the antecedent of $m$. According to (CR2), $\mu_{m_{4}}\left(d_{4}\right)=0.9>\varepsilon$, hence $d$ is covered by the conclusion of $m$. For another individual $z=$ "1212" which represents the rule "IF $\vartheta$ is $L$ AND $T_{r}$ is $M$ AND $\delta$ is $L$, THEN $\eta$ is $M$," $d$ is also covered by the antecedent of $z$, but not covered by the conclusion of $z$, because $\mu_{z_{4}}\left(d_{4}\right)=\mu_{2}(0.05)=$ $\mu_{M}(0.05)=0.1<\varepsilon$.

4) GA: Details of the GA are given as follows.

1) Generation of the initial population (steps 3-5 in Fig. 3). The size of each population is denoted as POPSIZE. All the rules in $R_{i}$ are copied into the initial population as part of the individuals. Additionally, other new individuals are randomly generated, with the values for each variable randomly generated from the value domain.

2) Fitness calculation (step 6 in Fig. 3). When a new population is generated, the fitness of each individual is computed from data $D_{i}$.

3) Crossover operator (step 7(b) in Fig. 3). Two individuals are chosen for crossover according to a crossover probability $\left(p_{\mathrm{c}}\right)$. If a randomly generated decimal is lower than or equal to $p_{\mathrm{c}}$, crossover will be conducted between these two individuals. For crossover between two individuals, a crossover position is randomly picked, and the segments of the two individuals after the crossover position are swapped. For example, for two individuals " 1111 " and " 2222 " that are selected for crossover, if the first position is picked, the two offspring generated by crossover are "2111" and "1222," respectively.

4) Mutation operator (step 7(d) in Fig. 3). To apply mutation on an individual, a position is randomly picked and the value in the selected position is randomly altered to another value in the domain. For example, for an individual " 1232 ," if the third position is picked, and a value " 0 " is randomly generated, then the offspring after mutation is "1202."

5) Repair operator (step 7(c) in Fig. 3). Since "0" may appear in individuals, an individual containing all " 0 "s in the positions represents a null rule and is invalid. Therefore, it is necessary to verify each individual. For an individual to represent a valid rule, there should be at least one nonzero value in the antecedent, and a nonzero value in the conclusion. A repair operator [27] is used to modify invalid individuals. If all the values in the antecedent are 0 , a nonzero value in the domain will be randomly introduced to a randomly picked position. If the value in the conclusion is 0 , it will be modified to a random nonzero value in $\{1,2,3\}$. Although this work and [27] apply GA with a repair operator to different problems, this work adopts the same principle as in [27]. In this work, the repair operator consists of 1) validating the antecedent and conclusion of an individual in the populations of rules and 2) correcting (repairing) the antecedent and/or conclusion of the rules. These tasks 
correspond to the fault detection and fault correction tasks, respectively, in [27]. In the validation of antecedents and conclusion of rules, the detection of an invalid rule triggers the application of the correction or repair of the rule. As noted in [27], doing so increases the number of generated individuals that form part of the valid search space (i.e., decreases the number of generated individuals that form part of the invalid search space), and including the repair operator in the GA is likely to increase its efficiency because empirical results in [27] showed that when their GA is implemented with the repair operator, it converged on the better solutions significantly faster than one without the repair operator.

6) Tournament selection method (step 7(g) in Fig. 3). In each selection, $q$ individuals are picked from the population randomly. The individual with the highest fitness among the $q$ individuals will be selected to be placed into the new population. The selection process will be repeated POPSIZE times.

7) Stopping criteria. Although a stopping criteria of 500 iterations is set for the GA presented in Fig. 3, experimental tuning has demonstrated that it generally converges rapidly, i.e., before 500 iterations is reached.

8) Parameter settings. Based on experimental tuning, the size of the population POPSIZE is set to 100, a tournament size of 7 is used, and the probabilities of crossover and mutation are 0.8 and 0.2 , respectively

\section{Simulation AND EXPERIMENTS}

A series of experiments was carried out using the testbed described below. Two kinds of experiments for evaluating and comparing EvEMDAs (MDAs with relaxed-criteria rules that are evolved using the evolutionary algorithm in Section IV) and EMDAs (MDAs with relaxed-criteria rules that are manually constructed) were carried out through stochastic simulations.

1) Testbed: To demonstrate the effectiveness of the procedure for evolving relaxed-criteria rules (Section IV), a testbed to simulate the negotiation activities of both EvEMDAs and EMDAs in a series of e-markets has been developed. Implemented using $\mathrm{C}++$, the testbed consists of an $e$-market place, a controller, and negotiation agents.

a) Controller: The controller generates negotiation agents, randomly assign values for their parameters (e.g., their roles as buyers or sellers, initial prices, reserve prices, deadlines, and $\lambda$ ), and simulates the entrance of agents to the e-marketplace following a uniform distribution.

b) e-market place: In an e-market, negotiation agents are either buyer agents $\left\{B_{1}, B_{2}, \ldots, B_{m}\right\}$ or seller agents $\left\{S_{1}, S_{2}, \ldots, S_{n}\right\}$. In each round, each $B_{i}$ or $S_{j}$ performs the following: a) decides whether to accept its trading parties' proposals following rules $R 1$ and $R 2$ specified in Section III-A, and b) if an agreement is reached, it leaves the e-market; otherwise, it generates its next proposal $P_{\mathrm{t}+1}$ following the specifications in Section II. For the purpose of simulation, a negotiation agent is either an MDA, an EMDA, or an EvEMDA.
TABLE II

INPUT DATA SOURCE

\begin{tabular}{l|c|c|c}
\hline \multicolumn{1}{c|}{ Input Data } & \multicolumn{3}{|c}{ Possible Values } \\
\hline \hline e-market type & Favorable & Balanced & Unfavorable \\
\hline $\begin{array}{l}\text { Characteristic: } \\
\text {-Buyer-seller } \\
\text { ratio }\end{array}$ & $\{1: 5\}$ & $\{1: 1\}$ & $\{5: 1,10: 1\}$ \\
\hline Setting: $P_{\text {buyer }}$ & $<0.5$ & 0.5 & $>0.5$ \\
\hline \multicolumn{3}{c}{$P_{\text {buyer }}:$ probability of an agent being a buyer } \\
\hline $\begin{array}{l}\text { Deadline } \\
\text { (No. of rounds) }\end{array}$ & \multicolumn{3}{|c}{$830-39,40-49,50-59,60-69,70-79,80-$} \\
\hline Strategy $\lambda$ & \multicolumn{3}{|c}{$1 / 10,1 / 3,1,2,3,10$} \\
\hline
\end{tabular}

TABLE III

Performance Measures

\begin{tabular}{|c|c|}
\hline Success Rate (SR) & $R_{\text {success }}=N_{\text {success }} / N_{\text {total }}$ \\
\hline $\begin{array}{l}\text { Expected Utility } \\
(E U)\end{array}$ & $\begin{array}{l}U_{\text {expected }}= \\
U_{\text {success }} \times R_{\text {success }}+U_{\text {fail }} \times\left(1-R_{\text {success }}\right)\end{array}$ \\
\hline $\begin{array}{l}\text { Negotiation } \\
\text { Speed (NS) }\end{array}$ & $N S=\sum_{i}^{N_{\text {success }}} n_{\text {rounds }}^{i} / N_{\text {success }}$ \\
\hline $\begin{array}{l}\text { Combined } \\
\text { Outcome }(\mathrm{CO})\end{array}$ & $C O=R_{\text {success }} \times U_{\text {success }} /$ Norm $_{\text {speed }}$ \\
\hline$\overline{N_{\text {total }}}$ & Total number of agents \\
\hline$N_{\text {success }}$ & No. of agents that reached consensus \\
\hline$N_{\text {total }}-N_{\text {success }}$ & No. of agents that did not reach consensus \\
\hline$U_{\text {success }}$ & $\begin{array}{l}\text { Average utility of agents that reached } \\
\text { consensus }\end{array}$ \\
\hline$U_{\text {fail }}=0$ & $\begin{array}{l}\text { Average utility of agents that did not reach } \\
\text { consensus }\end{array}$ \\
\hline$n_{\text {rounds }}^{i}$ & $\begin{array}{l}\text { No. of negotiation rounds that agent } i \text { used to } \\
\text { reach a consensus }\end{array}$ \\
\hline$\tau^{i}$ & Agent i's deadline \\
\hline $\begin{array}{l}\text { Norm }_{\text {speed }} \\
\text { (Normalized } \\
\text { speed). }\end{array}$ & Norm $_{\text {speed }}=\sum_{i}^{N_{\text {success }}}\left(n_{\text {rounds }}^{i} / \tau^{i}\right) / N_{\text {success }}$ \\
\hline
\end{tabular}

2) Objectives and Motivations: The two kinds of experiments are designed to explore the influence of market dynamics on the performance of EvEMDAs and EMDAs in a series of e-markets. By comparing EvEMDAs with EMDAs, one can study the possible benefits of allowing EvEMDAs to have the flexibility of evolving their relaxed-criteria fuzzy rules to adapt to different trading environments and to improve their performance. By simulating the negotiation activities of EvEMDAs (and also EMDAs) in a series of e-markets, one can study the potential self-improving property of EvEMDAs.

In the first (respectively, second) type of experiments, buyer agents are EvEMDAs (respectively, EMDAs) and seller agents are MDAs. The reason that seller agents are MDAs is explicated as follows. The MDA strategy, FDC, and relaxed-criteria rules are all designed for both buyer and seller agents; hence, without loss of generality, it suffices to demonstrate the properties of EvEMDAs from the perspective of buyer agents. The experimental setting used here is based on a common assumption in microeconomics, namely ceteris paribus [28]. By making a ceteris paribus assumption, the effect of a particular factor can be analyzed by holding all other factors constant. Since the purpose is to only compare EvEMDAs and EMDAs from 


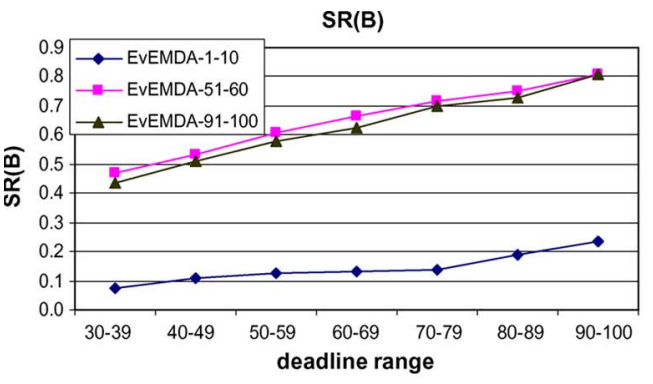

(a)

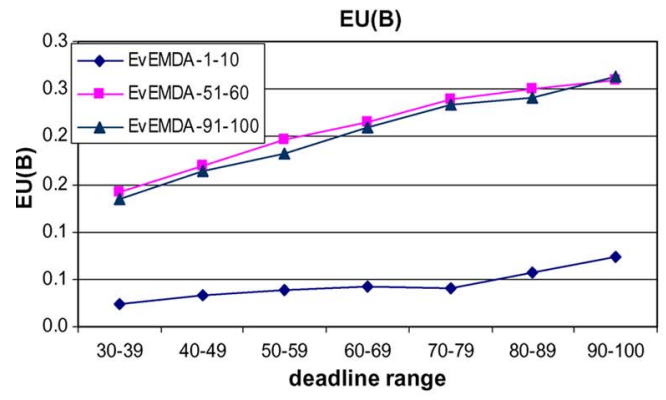

(c)

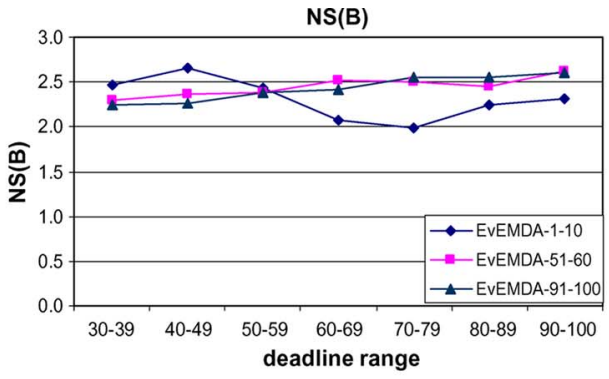

(b)

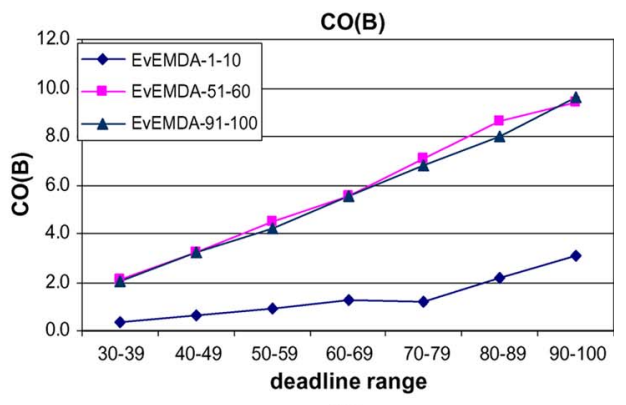

(d)

Fig. 4. Buyer-seller ratio $10: 1$

the perspective of buyer agents, it seems prudent to avoid any possible influence on the negotiation outcomes when seller agents make relaxations. Hence, in both types of experiments, seller agents are programmed as MDAs because MDAs are not designed to relax their bargaining criteria.

3) Experimental Settings: To demonstrate the selfimproving property of EvEMDAs, the negotiation activities of EvEMDAs (and EMDAs) are simulated in a series of 100 e-markets. Whereas the same set of fuzzy rules for EMDAs is used throughout the entire series of 100 markets, the procedure in Section IV is used to evolve the set of fuzzy rules of EvEMDAs in between two e-markets. As shown in Table II, there are three input parameters to the testbed i.e., the $e$-market type, negotiation deadline, and strategy $\lambda$.

There are three types of e-markets: favorable, balanced, and unfavorable, and they are characterized by the ratio of buyer to seller agents. In each experiment, after every ten rounds, there is a 0.5 probability of generating an agent, and the probability $P_{\text {buyer }}$ of the newly generated agent being a buyer (see Table II) determines the e-market type. From a buyer agent's perspective, for a favorable (respectively, an unfavorable) e-market, an agent enters an e-market with a lower (respectively, higher) $P_{\text {buyer }}$ and a higher (respectively, lower) probability of being a seller agent. Hence, in a favorable (respectively, an unfavorable) emarket, there is little (respectively, stiff) competition among buyer agents as there are more (respectively, fewer) sellers. In an (almost) balanced e-market, the ratio is simply $1: 1$ since there are (almost) equal number of buyers and sellers. In this paper, experiments are conducted for buyer-seller ratios $\{10: 1,5: 1,1: 1,1: 5\}$. Buyer-seller ratios such as $1: 10$ and $1: 100$ are not considered since the FDC and the relaxedcriteria rules are generally designed such that they are unlikely to be triggered in extremely favorable (or very favorable) emarkets. In extremely favorable (or very favorable) e-markets, there is a very high chance that an EvEMDA (respectively,
EMDA) will face little or no competition. Furthermore, with plenty of trading parties, there is also much higher chance that an agent will receive proposals from trading parties that are matching or even exceeding its own proposal. Whereas the experiments were carried out in (very) unfavorable e-markets (e.g., buyer-seller ratios such as 10:1 and 5:1), extremely unfavorable e-markets (e.g., buyer-seller ratio such as 100:1 and $1000: 1)$ are not considered. This is because it was noted in [21] that in extremely unfavorable trading environments, only at most one pair of buyer and seller can complete the deal, and the probability that an agent successfully completing a deal is inherently extremely low even if it relaxes its bargaining terms. For instance, for a 100: 1 buyer-seller ratio, at any round, there is only a probability of $1 / 100$ that a buyer can complete a deal, if there is at least one seller agent in the market.

For simulation purpose, an agent's deadline for completing a task is measured in number of rounds (time units). An agent's deadline is randomly generated from between 30 and 100 time units. From experimental tuning, it was found that: 1) for deadlines $<30$, very few agents can complete deals, and 2) for deadlines $>100$, there is plenty of time for trading and both EvEMDAs and EMDAs achieved very high success rates in reaching agreements (i.e., both EvEMDAs and EMDAs are generally not subject to much time pressure).

Whereas strategy $\lambda$ is randomly picked from $\{1 / 10,1 / 3,1$, $2,3,10\}$, and for simulation purpose an agent's proposals are made in the price range $[10,100]$.

4) Performance Measure: In the experimentation, 1) Success Rate, 2) Expected Utility, 3) Negotiation Speed, and 4) Combined Negotiation Outcome were used as performance measures for evaluating and comparing EvEMDAs and EMDAs in the 100 e-markets. These performance measures are defined in Table III.

5) Simulations: In the first set of simulations, EvEMDAs (buyer agents) adopting different values of $\lambda$ are subject 


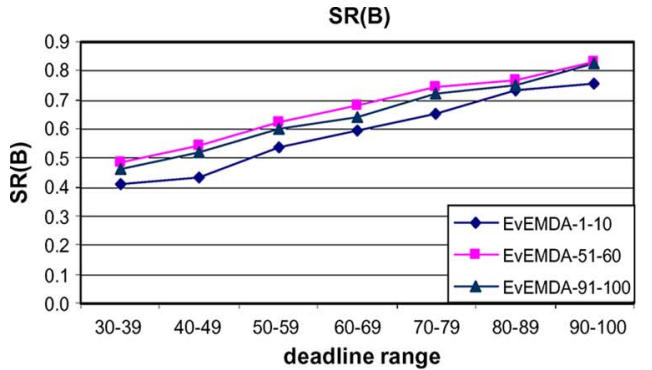

(a)

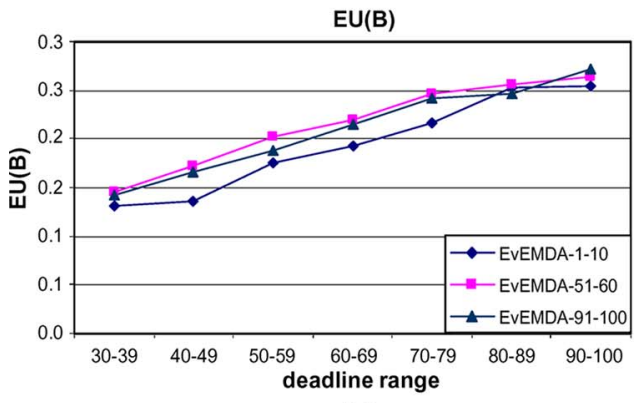

(c)

Fig. 5. Buyer-seller ratio $5: 1$.

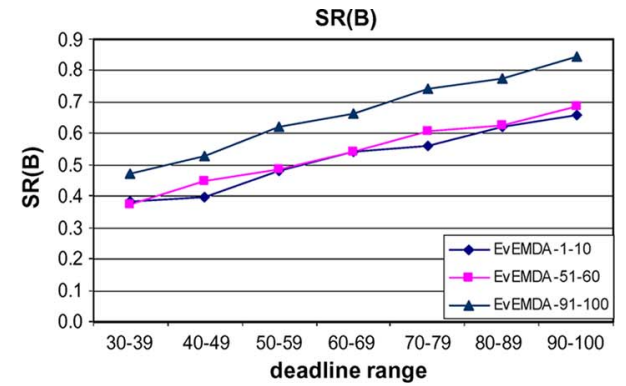

(a)

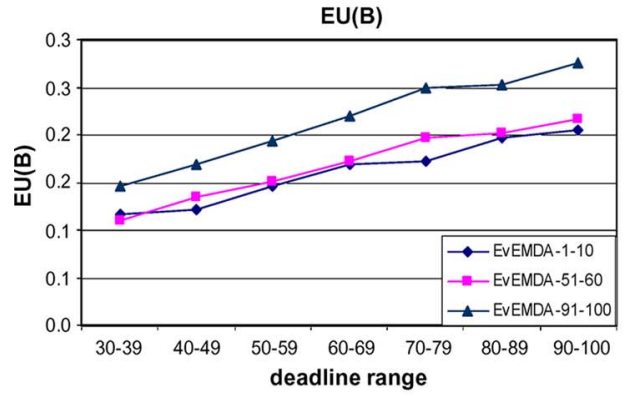

(c)

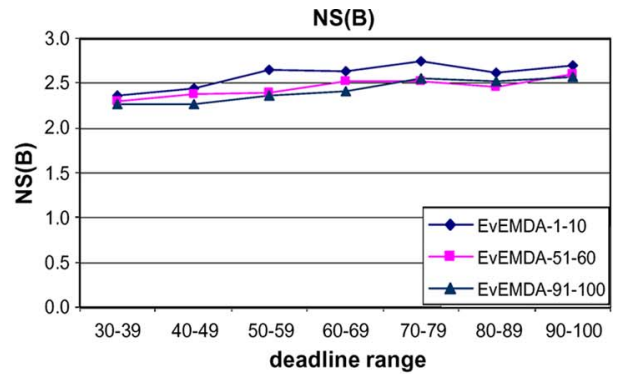

(b)

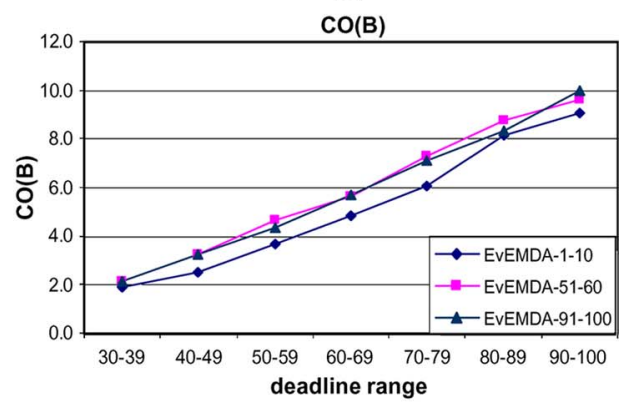

(d)

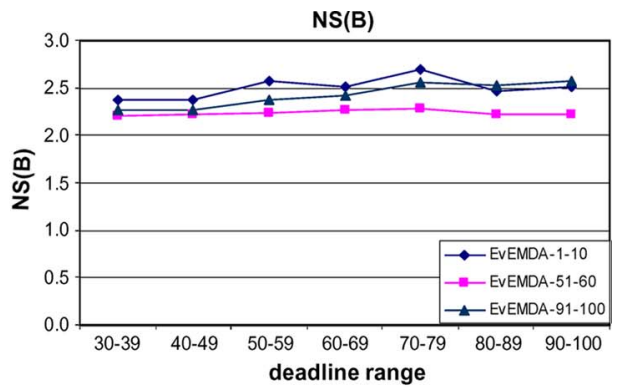

(b)

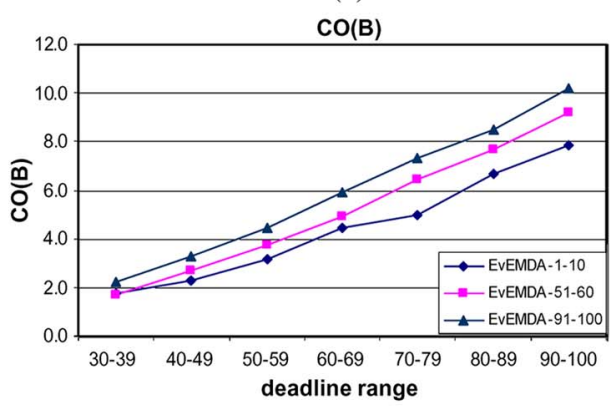

(d)

Fig. 6. Buyer-seller ratio $1: 1$.

to different deadlines and degrees of competition (different buyer-seller ratios) in 100 consecutive e-markets. In the second set of simulations, EMDAs (buyer agents) adopting different values of $\lambda$ are subject to the same settings. In both sets of simulations, the performance of EvEMDAs and EMDAs in 100 consecutive e-markets is recorded and the results are plotted in Figs. 4-8.

6) Results: Empirical results were obtained for all representative combinations of the input data (i.e., deadline $=$ $\{30-39,40-49,50-59,60-69,70-79,80-89,90-100\}$, buyer-seller ratio $=\{10: 1,5: 1,1: 1,1: 5\}$, both EvEMDAs and EMDAs adopting $\lambda=\{1 / 10,1 / 3,1,2,3,10\})$ in 100 consecutive e-markets. Due to space limitation, only the average performance of EvEMDAs and EMDAs in the first ten e-markets (markets 1-10), the middle ten e-markets (markets 51-60), and the last ten e-markets (markets 91-100) are plotted. For the four buyer-seller ratios $\{10: 1,5$ : $1,1: 1,1: 5\}, S R, E U, N S$, and $C O$ were plotted for the first, middle, and last ten e-markets for deadline $=$ $\{30-39,40-49,50-59,60-69,70-79,80-89,90-100\}$. Space limitation precludes having all the results being presented here. Whereas Figs. 4-7 show the performance of EvEMDAs in the first, middle, and last ten e-markets for buyer-seller ratios $\{10: 1,5: 1,1: 1,1: 5\}$, Fig. 8 shows 


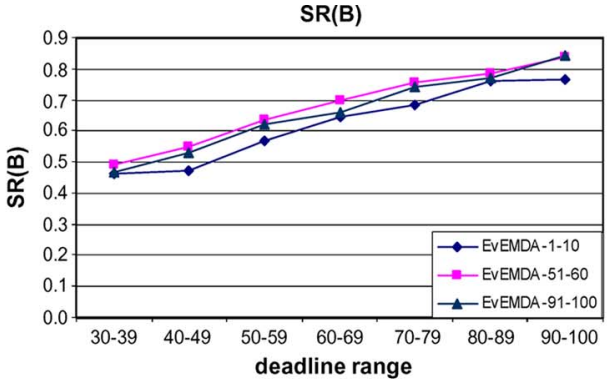

(a)

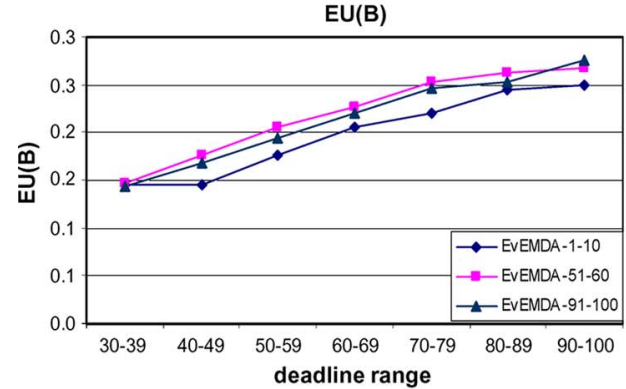

(c)

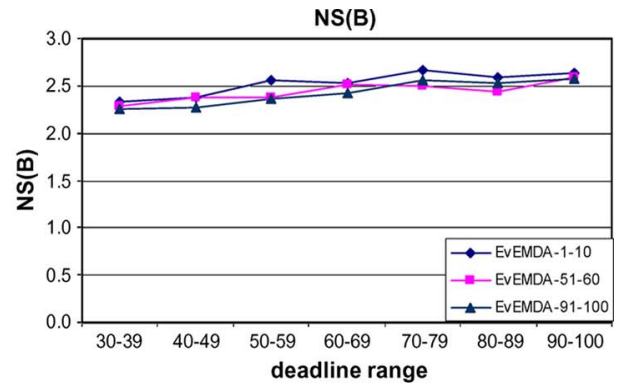

(b)

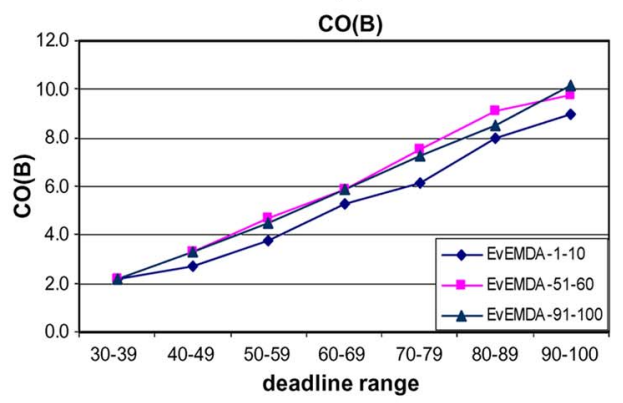

(d)

Fig. 7. Buyer-seller ratio $1: 5$.

the performance of EvEMDAs and EMDAs in the first and last ten e-markets for buyer-seller ratio $5: 1$. Results comparing EvEMDAs and EMDAs for $\{10: 1,1: 1,1: 5\}$ are similar to those of $5: 1$ and are omitted due to space limitation. In Figs. 4-7, the curves "EvEMDA-1-10," "EvEMDA-51-60," and "EvEMDA-91-100," respectively, represent the performance of EvEMDAs in the first, middle, and last ten e-markets. In Fig. 8, the curves "EvEMDA-1-10" ("EMDA-1-10") and "EvEMDA-91-100" ("EMDA-91-100") represent the corresponding performance of EvEMDAs (EMDAs) in the first and last ten e-markets.

7) Observation 1: It can be observed that the performance of EvEMDAs generally improved as they participated in the negotiations in more e-markets.

Fig. 4 shows the performance of EvEMDAs in very unfavorable e-markets (buyer-seller ratio 10:1). In Fig. 4, for the first ten e-markets (see "EvEMDAs-1-10"), the performance of $E v E M D A s$ is modest and NS fluctuated around 2.2 negotiation rounds. However, $N S$ stabilized in the middle and last ten e-markets. This is because in the first several e-markets, some of the manually designed rules were mainly used by EvEMDAs for relaxing their criteria. However, as EvEMDAs negotiated in more e-markets, new fuzzy rules were evolved, and it can be observed that both "EvEMDA-51-60" and "EvEMDA-91-100" clearly had higher $S R, E U$, and $C O$ than "EvEMDA-1-10." Consequently, it can be observed that the performance of EvEMDAs improved significantly as they negotiated in more unfavorable e-markets.

From Fig. 5 (buyer-seller ratio $5: 1$ ), it is observed that in an unfavorable e-market $E v E M D A s$ achieved higher $S R, E U$, and $C O$, and faster $N S$ in "EvEMDA-51-60" and "EvEMDA-91100 " than in "EvEMDA-1-10."

From Fig. 6 (buyer-seller ratio $1: 1$ ), it is observed that for a balanced e-market, $S R$ and $E U$ of EvEMDAs in "EvEMDA51-60" were slightly higher than those in "EvEMDA-1-10."
However, as EvEMDAs negotiated in more e-markets, it can be seen that $S R$ and $E U$ of EvEMDAs in "EvEMDA-91100 " improved significantly as compared to the $S R$ and $E U$ of EvEMDAs in "EvEMDA-51-60." From Fig. 6(d), it can be observed that the $C O$ of $E v E M D A s$ clearly improved for every deadline range as they negotiated in more e-markets. This result clearly shows the self-improvement property of EvEMDAs.

From Fig. 7 (buyer-seller ratio 1:5), it can be observed that in a favorable e-market, EvEMDAs achieved slightly better performance in $S R, E U, N S$, and $C O$ as they negotiated in more e-markets. However, the improvements were less significant than in the unfavorable and balanced e-markets. This is because in a favorable e-market, an $E v E M D A$ is less likely to relax their bargaining criteria to reach agreements.

8) Observation 2: EvEMDAs achieved better performance in terms of $S R, E U, N S$, and $C O$ than EMDAs.

From Fig. 8(a)-(d) (buyer-seller ratio $5: 1$ ), it can be seen that the $S R, E U$, and $C O$ of EvEMDAs in "EvEMDA-1-10" were clearly higher than those of EMDAs in "EMDA-1-10" for different deadline ranges, and EvEMDAs in "EvEMDA-110 " had clearly faster $N S$ than EMDAs in "EMDA-1-10" for all the deadline ranges. The results in Fig. 8(a)-(d) show that EvEMDAs outperformed EMDAs in the first ten e-markets.

From Fig. 8(e)-(h) (buyer-seller ratio 5:1), it can be observed that the $S R, E U$, and $C O$ of EvEMDAs in "EvEMDA91-100" were clearly higher than those of EMDAs in "EMDA91-100" for different deadline ranges, and EvEMDAs in "EvEMDA-91-100" had clearly faster NS than EMDAs in "EMDA-91-100" for all the deadline ranges. Hence, the results in Fig. 8(e)-(h) show that EvEMDAs outperformed EMDAs in the last ten e-markets.

9) Results Summary: In summary, for very unfavorable, unfavorable, balanced, and favorable e-markets, the results in Figs. 4-7 show that EvEMDAs generally achieved better 


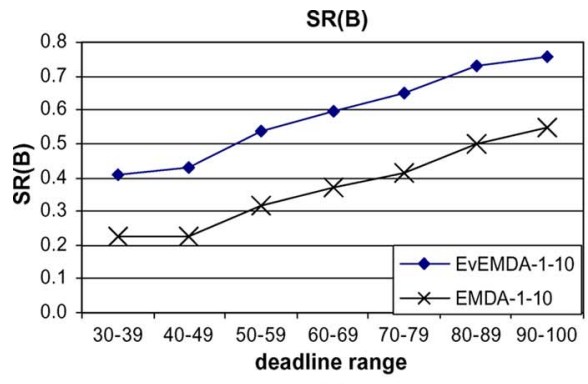

(a)

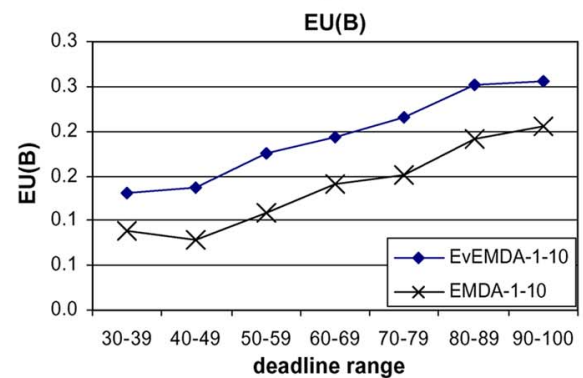

(c)

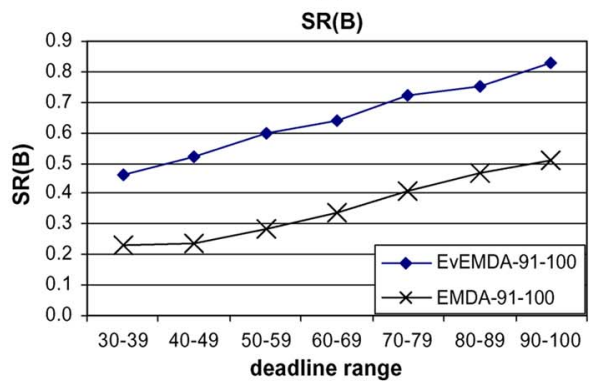

(e)

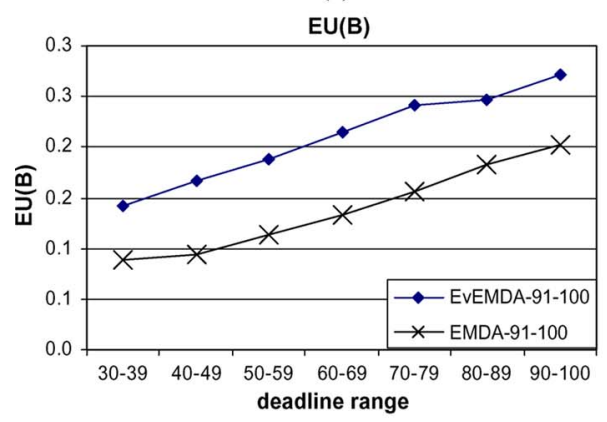

(g)

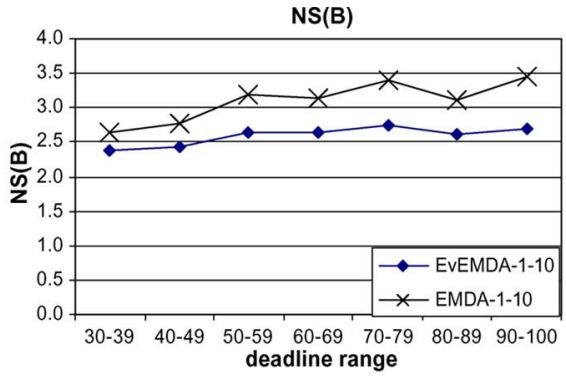

(b)

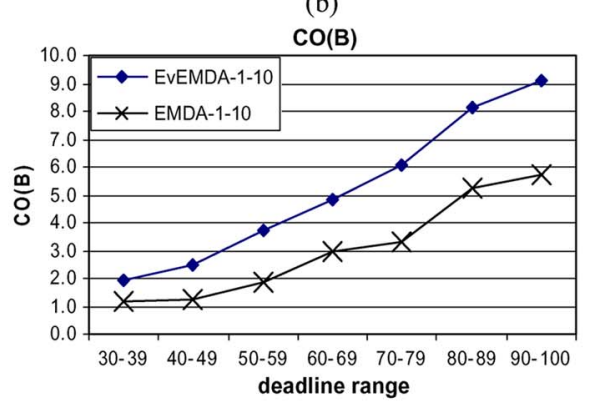

(d)

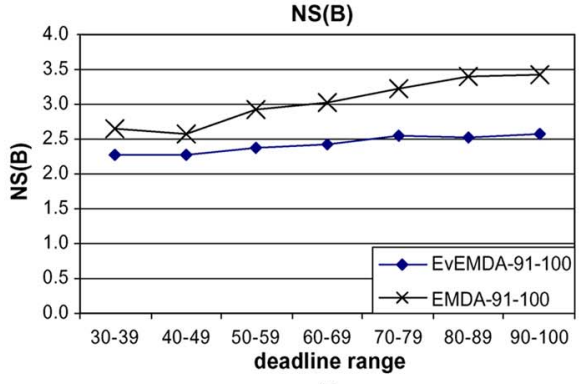

(f)

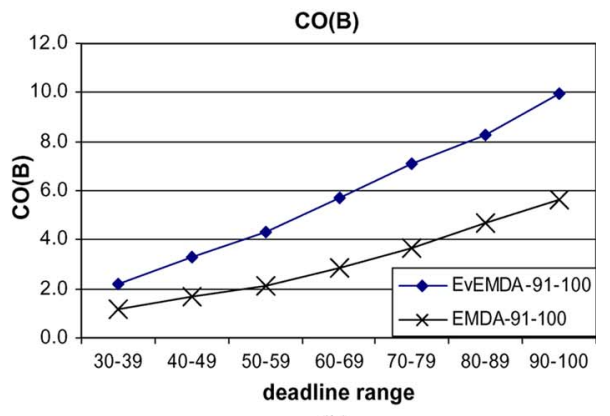

(h)

Fig. 8. Performance comparison between EvEMDAs and EMDAs (Buyer-seller ratio is $5: 1$ ).

performance as they evolved the relaxed-criteria fuzzy rules and participated in negotiations in more e-markets (observation 1). The results in Fig. 8 show that EvEMDAs generally achieved better performance than EMDAs both in the initial ten e-markets and as they participated in negotiations in more e-markets (observation 2).

\section{RELATED WORK}

This section reviews and discusses state-of-the-art negotiation agents that are designed with the flexibility to relax their trading conditions using fuzzy approaches (e.g., fuzzy constraint-based reasoning [29]-[33], fuzzy inference rules [34], and FDCs [5], [6], [21], [22]).
Fuzzy e-Negotiation Agents (FeNAs) [29]-[31] are fuzzy e-negotiation agents that adopt fuzzy constraint-based reasoning for bolstering multiissue negotiations. FeNAs negotiate by exchanging offers, and a consensus is reached when their private preferences, constraints, and objectives are satisfied. One of the distinguishing features of FeNAs is that preferences, priorities, and constraints are defined as fuzzy constraints (e.g., low price, high quality, short delivery time, and budget about $\$ 90)$. FeNAs's objectives may also be defined as soft objectives (e.g., an agent prefers to pay $\$ 200$ but is still happy with paying a little bit more). In FeNAs, preferences, constraints and objectives are represented by membership functions defining the degree of constraint satisfaction with possible solutions or agreements. During negotiation, the set of fuzzy 
constraints prescribes a fuzzy set of each FeNA's preferred potential solutions (individual area of interest). The possible joint solutions of negotiation (common interest area) are the intersection of individual interest areas. FeNAs' main goal is to find the joint solution within a common area of interest that maximizes the constraint satisfaction of the negotiating parties.

Lin et al. [32] and Lai and Lin [33] presented a problemsolving framework that uses fuzzy constraints for modeling multiissue multilateral negotiations for e-business agents. In [32] and [33], negotiation is formulated as a distributed fuzzy constraint satisfaction problem, which is represented as a set of fuzzy constraint networks (FCNs) that are connected by constraints. The objective is to determine whether a solution exists that can satisfy a specified number of constraints. In [32] and [33], each FCN is considered as an agent, and it contains a set of objects (issues) and fuzzy constraints (e.g., "High Amount," "Short Period"). Since an agent does not have a precise estimate of other agents' private information, each agent takes turn to pass messages to other agents to explore their information and search for a global solution. An order of information exchanges and a leadership token are defined to construct an interaction mechanism among the agents. In each round of coordination, the coordinating agent holding the leadership token first proposes its preferred solution according to its concession strategy which decreases its previous aggregated satisfaction value, or a tradeoff strategy to search for appropriate proposals. The proposed solution will be evaluated by other agents to determine whether it is satisfactory according to their own degrees of satisfaction. If the solution does not satisfy the constraints of any other agent, the coordinating agent passes the token to the next agent according to the predefined order. Then, the new coordinating agent will repeat the process of message passing. The procedure continues until either a consensus is reached or no other solution can be proposed. A consensus is reached only when a solution satisfies all the agents. Both [29]-[31] and [32], [33] modeled negotiation as a distributed fuzzy constraint satisfaction problem. The general difference is that while [29]-[31] deals with bilateral multiissue negotiations, [32], [33] deals with multilateral multiissue negotiations. Whereas [32] and [33] focused on finding a joint agreement that satisfies all constraints and maximizes the agents' aggregated degree of satisfaction, this work adopts a set of fuzzy rules to guide agents in relaxing their bargaining terms to enhance their chances of reaching agreements and reaching agreements more rapidly.

Wasfy and Hosni [35] introduced a model using a fuzzy logic approach to represent the strategic profiles in the negotiation process and to integrate several negotiation theories. In [35], a two-party multiissue negotiation situation was considered and simulated. Three moves were defined in their model: structural moves (alternating offers and counteroffers), communicative moves (attempt to change the negotiation power structure by informing the opponent of either the truth/deception about the preferences or intentions of the negotiator), and umpire moves (force changes in the properties of the other negotiation entities such as changing a negotiator's power as time passes). During negotiation, negotiators exchange communicative moves with their opponents to alter specific properties of the negotiation process. These properties are used to determine concessions in structural moves. In addition, umpire moves may be used to create time pressure on the negotiators by reducing their power as time expires and by forcing a deadline for reaching agreement. The process continues until an agreement is reached or the time for negotiation expires. In [35], the strategic profiles which represent a negotiator's natural willingness and unwillingness to concede are represented as a series of linear fuzzy trapezoidal shapes. The negotiation power (such as legitimacy, commitment, risk taking, time available) were also represented as fuzzy values in their simulations. Whereas [35] modeled negotiation power and (un)willingness to concede using fuzzy concepts, this work uses a set of fuzzy rules to guide agents in determining whether to reach agreements in multilateral negotiations.

Wu et al. [36] presented a fuzzy negotiation model for bilateral multiissue negotiations in e-commerce. Whereas this work adopts an alternating protocol, [36] adopted the monotonic concession protocols [1]. In [36], the acceptability for each issue was represented by a fuzzy value and their membership functions were normal distribution functions. The acceptability of an offer is determined by the aggregated acceptability for each of the issues with a weight assigned for each issue. A critical value was defined for each negotiation. During negotiation, (counter-)offers were exchanged between the buyer agent and the seller agent. When an agent's acceptability for its opponent's offer exceeds or equals the critical value, the negotiation ends with an agreement. Otherwise, the negotiator generates a new (counter-)offer or exits the negotiation without successfully completing a deal.

Closer to this work is the work of [34] that used fuzzy inference rules for determining the acceptance of an opponent's offer. Wang et al. [34] used a set of manually generated fuzzy rules for evaluating the incoming offer and the newly generated offer. If the acceptance of the incoming offer is greater than the acceptance of the (counter-)offer determined by the agent, an agreement is reached. Otherwise, the agent sends a newly generated offer to the opponent. However, if the acceptance of the incoming offer is less than the minimum acceptance of the agent, the incoming offer is rejected and negotiation is terminated. The exchange continued until an agreement is reached, or the maximum number of negotiation rounds or the maximum duration of negotiation is reached. One of the main differences between [34] and this work is that [34] adopted a fixed manually designed set of fuzzy rules while an evolving set of fuzzy rules is used in this paper. Furthermore, whereas [34] focused on modeling multiissue, bilateral negotiations involving a third-party-driven virtual marketplace, this work adopts fuzzy rules for relaxing bargaining terms in multilateral negotiations in which there is no third party mediation.

Meng and $\mathrm{Fu}$ [37] presented a negotiation model based on a fuzzy multiple criteria decision-making method for multiissue negotiation problems. In [37], negotiators' preferences (weights) of issues and evaluations of issues were represented as fuzzy variables with trapezoidal membership functions. Fuzzy linear weighted arithmetic average was used to obtain fuzzy evaluation of negotiators. Hamming gap is adopted to compute the similarity degree between the negotiation proposal 
and the ideal solution or the negative-ideal solution. A negotiator's satisfaction degree with the negotiation proposal was computed by the similarity degree between the proposal and the ideal solution and the similarity degree between the proposal and the negative-ideal solution. If all negotiators' satisfaction degrees are not less than the threshold of the satisfaction degrees, an agreement is reached and the negotiation is completed. Otherwise, the negotiators modify their proposals according to their evaluations, and negotiation continues until either an agreement is reached or the maximum number of sessions has been reached.

Sim and Wang [21] enhanced the design of MDAs (Section II) using a set of manually generated fuzzy rules to determine when they should relax their bargaining terms in the hope of having a higher chance of reaching a consensus. Augmented with an FDC [21], Sim's enhanced MDA (EMDAs) are programmed to slightly relax their bargaining terms in the face of intense pressure (e.g., urgent need to acquire a resource, or facing fast approaching deadlines). Since notions such as "very slight" difference in proposals, "strong" competition, and "fast" approaching deadline are vague, an FDC together with a set of 16 fuzzy rules were used in [21] to guide EMDAs in making decision when relaxing their aspirations. In relaxing its bargaining terms, an EMDA is influenced by factors such as degree of competition $(\vartheta)$, and its eagerness $(\varepsilon)$. $\varepsilon$ represents how urgent it is for an EMDA to acquire a resource before a deadline [21] and $\varepsilon=1 / \lambda$, because an EMDA that is more (respectively, less) eager to reach a consensus will adopt a strategy with a smaller (respectively, larger) value of $\lambda$. Both $\vartheta$ and $\varepsilon$ form the antecedents of the fuzzy rules while the amount of relaxation $\eta$ is the consequent. Whereas $\vartheta$ and $\varepsilon$ are the inputs to the FDC, $\eta$ represents the amount that an EMDA would relax its bargaining terms in a given situation (the output of the FDC). This work differs significantly from [21] in the following ways.

1) Whereas the set of 16 fuzzy rules in [21] was generated manually, the set of 27 fuzzy rules in this paper is evolved to improve the performance of agents as they negotiate in more e-markets.

2) While two relaxation criteria degree of competition $(\vartheta)$ and eagerness $(\varepsilon)$ were used in [21], three relaxation criteria a) degree of competition $(\vartheta)$, b) time pressure $\left(T_{r}\right)$, and c) the relative distances from trading parties' proposals $(\delta)$ are used in this work.

Since an agent that is more (respectively, less) urgently in need to acquire a resource before a deadline would have selected a strategy with a smaller (respectively, larger) value of $\lambda$, in this work, the author prefers to replace $\varepsilon$ with two relaxation criteria: time pressure and distances from trading parties. Time pressure is a more crucial factor when an agent considers whether to relax its bargaining terms because an agent has to complete negotiation before its deadline to successfully acquire a resource. Considering the distances between the proposal of an agent and all the proposals of its trading parties is essential because if its own proposal is relatively close to a trading party's proposal and relatively far from proposals of all other trading parties, it seems prudent for an agent to relax its bargaining terms to reach an agreement more rapidly rather than to continue with the negotiation.

The work in [21] was subsequently adapted to automated negotiation in grid resource management. In Sim's relaxed-criteria G-negotiation protocol [5], [6], market-driven $G$-negotiation agents representing resource providers and consumers are programmed to slightly relax their bargaining criteria under intense pressure (e.g., when a consumer has a higher demand for resources) with the hope of enhancing their chance of successfully acquiring resources. A consumer agent and a provider agent are both designed with an FDC: $F D C-C$ and $F D C$ - $P$, respectively. Two sets of relaxation criteria (for consumers and providers, respectively) that are specific to grid resource management are used as inputs to $F D C-C$ and $F D C-P$, respectively.

Two criteria that can influence a consumer agent's decision in the amount of relaxation of bargaining terms are: 1) recent statistics in failing/succeeding in acquiring resources called failure to success ratio $\left(f s_{t}\right)$ and 2 ) demand for computing resources called demand factor $\left(d f_{t}\right)$. If a consumer agent is less successful in acquiring resources recently to execute its set of tasks, it will be under more pressure to slightly relax its bargaining criteria with the hope of completing a deal. Furthermore, if it has a greater demand for computing resources it is more likely to be under more pressure to slightly relax its bargaining criteria. Both $f s_{t}$ and $d f_{t}$ are inputs to $F D C-C$, which a consumer agent uses to determine $\eta$ (its amount of relaxation) [5], [6].

Two criteria that can influence a provider agent's decision are: 1) the amount of the provider's resource(s) being utilized (i.e., the utilization level $\left(u l_{t}\right)$ ) and 2) recent requests from consumers for resources (i.e., called the request factor $\left(r f_{t}\right)$ ). If more of its resources are currently being used to execute its own tasks or have already been leased to other consumers, then a provider is less likely to slightly relax its bargaining terms. If there are fewer recent demands from consumers to lease its resources, a provider is more likely to slightly relax its bargaining criteria since it is under more pressure to trade its idle resources. Both $u l_{t}$ and $r f_{t}$ are inputs to $F D C$ - $P$, which a provider agent uses to determine $\eta[5],[6]$.

Unlike the two sets of fuzzy rules for $F D C-C$ and $F D C-P$ in [5] and [6] that were manually generated, the fuzzy rules in this work are evolved as agents negotiate in more e-markets.

\section{CONCLUSION}

The novelty and significance of this work are that (to the best of the author's knowledge) it is one of the earliest works that design negotiation agents that not only use fuzzy rules to relax bargaining criteria, but also evolve new relaxed-criteria fuzzy rules to improve their negotiation outcomes as they participate in negotiations in more e-markets.

The contributions of this work are detailed as follows.

1) Compared with other related works discussed in Section VI that adopt fuzzy approaches for building negotiation agents, this work (to the best of the author's knowledge) is the earliest work that adopts an evolutionary 
fuzzy approach for constructing adaptive and selfimproving negotiation agents operating in a series of e-markets.

2) An evolutionary algorithm for adapting and evolving relaxed-criteria fuzzy rules was developed. Using the negotiation outcomes of agents in each e-market as data sets, the algorithm learns effective relaxed-criteria negotiation rules that would potentially enhance the performance of MDAs as they negotiate in subsequent markets.

3) A new FDC was designed and implemented with the following novel features.

a) A new set of relaxation criteria was formulated, and the criteria time pressure $\left(T_{r}\right)$ and the relative distances from trading parties' proposals $(\delta)$ were used in place of eagerness $(\varepsilon)$ in [21].

b) While the $F D C$ in [21] has two inputs and consults a rule base with 16 fuzzy rules, the new FDC in this paper has three inputs and consults a rule base with 27 fuzzy rules.

c) More importantly, while the set of 16 fuzzy rules in [21] was constructed manually and used throughout the entire life-span of agents in [21], the set of 27 fuzzy rules in this work is evolved to improve the performance of agents as they negotiate in more e-markets.

4) A testbed to demonstrate the effectiveness of evolving relaxed-criteria fuzzy rules of negotiations was developed. Whereas previous testbeds in [5], [6], and [21] consisted of only MDAs and EMDAs, the testbed in this paper is used to compare the performance of EvEMDAs with EMDAs (see Section V).

5) Empirical results (Section V) show that: a) EvEMDAs generally outperformed EMDAs in terms of $S R, E U$, $N S$, and $C O$ and b) the negotiation outcomes of EvEM$D A s$ generally improved as they negotiated in more emarkets. These results provide evidence to show that evolutionary fuzzy approaches are appropriate paradigms for designing adaptive and self-improving negotiation agents operating in a series of e-markets. It is reminded that whereas $S R$ and $E U$ were used as performance measures for comparing MDAs and EMDAs in only one e-market in [21], the experiments in this paper compared the performance of EvEMDAs and EMDAs in terms of $S R, E U, N S$, and $C O$ in a series of 100 e-markets.

Finally, the author acknowledges that although this work adopts an evolutionary fuzzy approach for developing agents in multilateral single-issue negotiations, in its present form, this paper does not deal with multiissue negotiations like some of the related work (e.g., [29]-[37]) discussed in Section VI. Hence, extending this work to include multiissue negotiations, perhaps, by adopting some of the techniques of fuzzy constraint-based reasoning in [29]-[33] is among the list of agendas for future work. Additionally, in its present form, the fuzzy membership functions in this paper are predetermined and fixed for the fuzzy variables. Other possible enhancements of this work may include 1) automatic and dynamic tuning of the membership functions by GAs and 2) adopting trapezoidal membership functions (rather than those in Fig. 1) for assigning memberships to the linguistic terms ( $\mathrm{L}, \mathrm{M}$, and $\mathrm{H}$ ).

\section{ACKNOWLEDGMENT}

The author would like to thank the Editor-in-chief, the Associate Editor, and anonymous referees for their comments and suggestions. The author would also like to thank Y. Y. Guo for carrying out the implementation and experimentations.

\section{REFERENCES}

[1] J. Rosenschein and G. Zlotkin, Rules of Encounter: Designing Conventions for Automated Negotiation Among Computers. Cambridge, MA: MIT Press, 1994

[2] R. Wolski et al., "Grid resource allocation and control using computational economies," in Grid Computing - Making the Global Infrastructure a Reality, F. Berman, A. Hey, and G. Fox, Eds. New York: Wiley, 2003, pp. 747-771.

[3] K. M. Sim, "G-commerce, market-driven G-negotiation agents and grid resource management," IEEE Trans. Syst., Man, Cybern. B, Cybern., vol. 36, no. 6, pp. 1381-1394, Dec. 2006.

[4] K. M. Sim, "A survey of bargaining models for grid resource allocation," ACM SIGECOM: E-Commerce Exchange, vol. 5, no. 5, pp. 22-32, Jan. 2006.

[5] K. M. Sim and K. F. Ng, "A relaxed-criteria bargaining protocol for grid resource management," in Proc. 6th IEEE Int. Symp. CCGRIDW, Singapore, May 2006, p. 5.

[6] K. M. Sim and K. F. Ng, "Relaxed-criteria negotiation for G-commerce," in Proc. Business Agents and the Semantic Web Workshop, held in conjunction with the 5th Int. Joint Conf. Auton. Agents and Multi-Agent Syst., Hakodate, Japan, May 2006, pp. 53-61.

[7] J. F. Nash, "The bargaining problem," Econometrica, vol. 18, no. 2, pp. $155-162$, Apr. 1950.

[8] M. J. Osborne and A. Rubinstein, Bargaining and Markets. New York: Academic, 1990.

[9] A. Rubinstein, "A bargaining model with incomplete information about time preferences," Econometrica, vol. 53, no. 5, pp. 1151-1172, Sep. 1985.

[10] A. Rubinstein, "Perfect equilibrium in a bargaining model," Econometrica, vol. 50, no. 1, pp. 97-109, Jan. 1982.

[11] J. Harsanyi, Rational Behavior and Bargaining Equilibrium in Games and Social Situations. Cambridge, U.K.: Cambridge Univ. Press, 1977.

[12] A. Muthoo, Bargaining Theory With Applications. Cambridge, U.K.: Cambridge Univ. Press, 1999.

[13] T. Sandholm and N. Vulkan, "Bargaining with deadlines," in Proc. Nat. Conf. AAAI, Orlando, FL, 1999, pp. 44-51.

[14] A. Chavez and P. Maes, "Kasbah: An agent marketplace for buying and selling goods," in Proc. 1st Int. Conf. Practical Appl. Intell. Agents MultiAgent Technol., London, U.K., 1996, pp. 159-178.

[15] P. Faratin et al., "Negotiation decision functions for autonomous agents," Int. J. Robot. Auton. Syst., vol. 24, no. 3, pp. 159-182, 1998.

[16] A. Lomuscio et al., "A classification scheme for negotiation in electronic commerce," Int. J. Group Decis. Negot., vol. 12, no. 1, pp. 31-56, 2003.

[17] N. R. Jennings et al., "Automated negotiation: Prospects, methods and challenges," Int. J. Group Decis. Negot., vol. 10, no. 2, pp. 199-215, 2001.

[18] K. M. Sim, "A market-driven model for designing negotiation agents," Comput. Intell., vol. 18, no. 4, pp. 618-637, 2002.

[19] K. M. Sim and C. Y. Choi, "Agents that react to changing market situations," IEEE Trans. Syst., Man, Cybern. B, Cybern., vol. 33, no. 2, pp. 188-201, Apr. 2003.

[20] K. M. Sim, "Equilibria, prudent compromises, and the "waiting' game," IEEE Trans. Syst., Man, Cybern. B, Cybern., vol. 35, no. 4, pp. 712-724, Aug. 2005.

[21] K. M. Sim and S. Y. Wang, "Flexible negotiation agent with relaxed decision rules," IEEE Trans. Syst., Man, Cybern. B, Cybern., vol. 34, no. 3, pp. 1602-1608, Jun. 2004.

[22] K. M. Sim, "Negotiation agents that make prudent compromises and are slightly flexible in reaching consensus," Comput. Intell., vol. 20, no. 4, pp. 643-662, Nov. 2004.

[23] M. Wooldridge, An Introduction to Multiagent Systems. Hoboken, NJ: Wiley, 2002. 
[24] G. J. Klir, Fuzzy Set and Fuzzy Logic: Theory and Application. Englewood Cliffs, NJ: Prentice-Hall, 1995.

[25] T. J. Ross, Fuzzy Logic With Engineering Applications. New York: McGraw-Hill, 1995

[26] R. F. Roberto et al., "Discovering fuzzy classification rules with genetic programming and co-evolution," in Proc. 5th Eur. Conf. Principles Data Mining Knowl. Discov., 2001, pp. 314-325.

[27] G. Mitchell et al., "GeneRepair-A repair operator for genetic algorithms, late-breaking paper," in Proc. Genetic Evol. Comput. Conf., Chicago, IL, Jul. 2003, pp. 235-239.

[28] D. Salvatore, Microeconomics Theory and Applications. Reading, MA: Addison-Wesley, 1997.

[29] R. Kowalczyk, "Fuzzy e-negotiation agents," Soft Comput.-A Fusion of Foundations, Methodologies and Applications, vol. 6, no. 5, pp. 337-347, Aug. 2002.

[30] R. Kowalczyk and V. Bui, "FeNAs: A fuzzy e-negotiation agents system," in Proc. IEEE/IAFE/INFORMS Conf. CIFEr, New York, 2000, pp. 26-29.

[31] R. Kowalczyk and V. Bui, "On fuzzy e-negotiation agents: Autonomous negotiation with incomplete and imprecise information," in Proc. 11th Int. Conf. Database Expert Syst. Appl., 2000, pp. 1034-1038.

[32] M. Lin, K. R. Lai, and T. J. Yu, "Fuzzy constraint-based agent negotiation," J. Comput. Sci. Technol., vol. 20, no. 3, pp. 319-330, May 2005.

[33] K. R. Lai and M. W. Lin, "Modeling agent negotiation via fuzzy constraints in e-business," Comput. Intell., vol. 20, no. 4, pp. 624-642, Nov. 2004.

[34] X. Wang, X. Shen, and N. D. Georganas, "A fuzzy logic based intelligent negotiation agent (FINA) in e-commerce," in Proc. IEEE Can. Conf. Electr. Comput. Eng., Ottawa, ON, Canada, May 2006, pp. 276-279.

[35] A. M. Wasfy and Y. A. Hosni, "Two-party negotiation modeling: An integrated fuzzy logic approach," Group Decis. Negot., vol. 7, no. 6, pp. 491-518, Nov. 1998.
[36] Y. Wu, J. Lu, and F. Yan, "A fuzzy negotiation model of e-commerce and its implementation," in Proc. Technol. Manag. Global Future, PICMET, Jul. 2006, vol. 3, pp. 1180-1185.

[37] B. Meng and W. Fu, "A negotiation model based on fuzzy multiple criteria decision making method," in Proc. 4th Int. Conf. Comput. Inf. Technol., Sep. 2004, pp. 1039-1044.

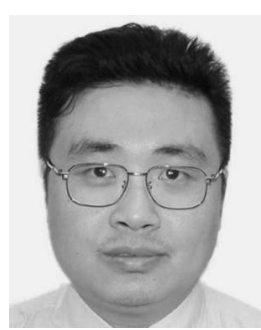

Kwang Mong Sim received the B.Sc. degree (Hon.) (summa cum laude) from the University of Ottawa, Ottawa, ON, Canada and the M.Sc. and Ph.D. degrees from the University of Calgary, Calgary, $\mathrm{AB}$, Canada.

Currently, he is with the Hong Kong Baptist University, Kowloon Tong, Hong Kong. He has served as a referee for several national research grant councils including the National Science Foundation, USA, and as Area Chair, Session Chairman, and Program Committee Member in many conferences. He has supervised over 30 researchers/graduate students.

Dr. Sim is an Associate Editor for the IEEE TRANSACTIONS ON SYSTEMS, MAN, AND CYBERNETICS-PART C, and the International Journal of Applied Systemic Studies, and an editorial/advisory board member of the International Journal of Hybrid Intelligent Systems, the System and Information Sciences Notes, and The Open Cybernetics and Systemics Journal. He is also the Guest Editor of five (IEEE) journal special issues in agent-based grid computing and automated negotiation, including a special issue on Grid Resource Management in the IEEE Systems JouRnAL (IEEE Systems Council). 